\title{
Higher Order Coercive Inequalities
}

\author{
Yifu Wang $^{1}$ (D) . Boguslaw Zegarlinski ${ }^{1}$ (i)
}

Received: 5 March 2021 / Accepted: 17 June 2021 / Published online: 13 July 2021

(C) The Author(s) 2021

\begin{abstract}
We study the higher order $q$ - Poincaré and other coercive inequalities for a class probability measures satisfying Adam's regularity condition.
\end{abstract}

Keywords Higher order Poincaré · Tight Orlicz-Sobolev inequalities · Regular probability measures · Higher order minimizers · Statistical polynomials · Equivalence of norms · Decay to equilibrium

\section{Introduction}

Studies of coercive inequalities for probability measures, in particular including Poincaré and Log-Sobolev type inequalities and their applications, has a long and interesting history see e.g. [4, 6, 13, 16-22]. Despite enormous progress in this area there is still a lot of widely open and challenging problems in particular in relation to analysis on Lie groups and infinite dimensional analysis, for some starting pointers in this direction the reader could consult e.g. [7, 23, 29-31]. In this paper we study in a systematic way the higher order inequalities in functional spaces associated to a probability measure. In case of Lebesgue measure an extensive literature exists see e.g. [38] and references therein. For probability measures first work done in the direction of higher order bounds is [33] with more extensive development achieved in [1]. In particular in the latter work [1], the author considered a class of probability measures for which the logarithm of density of the probability measure was given as a function of regularised distance in $\mathbb{R}^{n}$ and satisfied some pointwise regularity conditions. The coercive inequalities obtained in both papers were defective in the sense that besides the terms containing higher order derivatives it contained also terms dependent on $L_{p}$ norms. Expanding on techniques developed in [23], in the present paper we prove tight coercive inequalities.

To this end we study first higher order Poincaré inequalities for a class of probability measures containing those considered in [1]. Although getting optimal constants is currently

The first author was supported by the Imperial College Scholarship

Boguslaw Zegarlinski

b.zegarlinski@imperial.ac.uk

Extended author information available on the last page of the article. 
challenging for higher order inequalities, we provide a constructive method to get some estimates of them.

Later we use the higher order Poincaré inequalities to obtain tight coercive inequalities in the form of bounds from below on $L_{p}$ norms of higher order derivatives of a function by suitable Orlicz norms of the function with removed part which is nullified by the higher order derivatives. Unlike in case of Log-Sobolev inequality we do not have direct relation with tight coercive inequalities involving suitable functionals (as e.g. in [8-12, 14, 19, 32]). Thus in our context, in order to get a generalisation of Holley-Stroock perturbation lemma [24] it is more natural to consider norm dependent minimizer. We show that in some sense they are equivalent to the classical ones, but they are of other potential interest too if one would like to study concentration phenomena in various functional spaces.

Later, by developing further our techniques, we obtain quantitative estimates on the constants in the Adam's bounds of [1]. This allows us to show equivalence of $W_{k, p}(\mu)$ norms and norms related to the Markov generator associated to the natural Dirichlet form with the given probability measure. We remark that an extensive literature exists in relation to first order bounds with use of the Riesz transform see e.g. [5, 13, 34] and references therein. To get estimates of higher order some more effort was necessary.

Finally at the end of the paper we discuss decay to equilibrium properties on various spaces. The motivation for this study for us comes from the works [25-27, 36, 37], where various applications of higher gradient estimates were exploited in the infinite dimensional setting. One may hope that in the future further development of methods based on higher order estimates may help in better understanding difficult problems of decay to equilibrium for Markov semigroups in infinite dimensional context.

\section{Adams Inequalities}

Let $\lambda$ denotes the Lebesgue measure on $\mathbb{R}^{n}$. With a twice differentiable real function $U$ satisfying

we define

$$
0<\int e^{-U} d \lambda<\infty
$$

$$
d \mu \equiv e^{-U} d \lambda
$$

We say that $U$ is locally bounded if it is bounded on any ball $B(0, r)$ with radius $r \in$ $(0, \infty)$ centered at the origin.

In [1] the following condition was explored.

Adams Regularity Conditions $\exists \varepsilon, C \in(0, \infty)$

$$
\sum_{|\alpha|=2}\left|\nabla^{\alpha} U\right| \leq C(1+|\nabla U|)^{2-\varepsilon} \text {. }
$$

For $\alpha \equiv\left(\alpha_{j} \in \mathbb{Z}^{+}\right)_{j=1, . ., n}$ we set

$$
|\alpha| \equiv \sum_{j=1, . ., n} \alpha_{j}
$$

and denote $\nabla^{\alpha} \equiv \prod_{j=1, \ldots, n} \partial_{j}^{\alpha_{j}}$. For $m \in \mathbb{N}$ and $p \geq 1$, define

$$
\|f\|_{m, p}^{p} \equiv\|f\|_{m, p, \mu}^{p} \equiv \sum_{\alpha:|\alpha| \leq m} \mu\left(\left|\nabla^{\alpha} f\right|^{p}\right)
$$


and let $W_{m, p}(\mu)$ denote the closure of compactly supported smooth functions $\mathcal{C}_{0}^{\infty}$ with respect to this norm.

Let $A$ be a nonnegative nondecreasing function on $\mathbb{R}^{+}$satisfying $\Delta_{2}$ condition, i.e. with some $K_{0} \in \mathbb{R}^{+} \forall t \in \mathbb{R}^{+}$

$$
A(2 t) \leq K_{0} A(t)
$$

and define

$$
\Phi_{A, p}(t) \equiv|t|^{p} A\left(\log ^{*}|t|\right),
$$

with $\log ^{*} t \equiv \max \{1, \log (t)\}$.

Theorem 1 (Adams'JFA1979, Theorem 1) If U satisfies the (ARC) regularity condition and if, for some $a \in(0, \infty)$, the following inequality holds

$$
A(U(x)) \leq a(1+|\nabla U|)^{m p}, \quad \text { a.e., }
$$

then there exists a constant $K \in(0, \infty)$ such that for all $f \in W_{m, p}(\mu)$,

$$
\mu \Phi_{A, p}(f) \leq K\left(\|f\|_{m, p}^{p}+\Phi_{A, p}\left(\|f\|_{p}\right)\right) .
$$

Lemma 1 (Adams'JFA1979, Lemma B) There exists a constant $\tilde{K}$ such that for every $f \in W_{m, p}(\mu)$,

in particular

$$
\int|f|^{p}(1+|\nabla U|)^{m p} d \mu \leq \tilde{K}\|f\|_{m, p}^{p}
$$

$$
\int|f|^{p}|\nabla U| d \mu \leq \tilde{K}\|f\|_{1, p}^{p}
$$

Proof In order to get some possibly useful information about the constants we provide here selfcontained arguments following [23]. First of all from Leibnitz rule for the gradient we have

$$
(\nabla f) e^{-U}=\nabla\left(f e^{-U}\right)+(\nabla U) f e^{-U}
$$

Multiplying both sides by the subunit vector $\nabla d$, with $d$ being a distance function which is regularised on small distances, integrating with the Lebesgue measure and performing integration by parts on the right hand side, one obtains

$$
\int \nabla d \cdot \nabla f d \mu=\int f(\nabla d \cdot \nabla U-\Delta d) d \mu
$$

Assuming

$$
\nabla d \cdot \nabla U-\Delta d \geq|\nabla U|-D
$$

for some $D \in(0, \infty)$ and using our assumption $|\nabla d| \leq 1$, we get

$$
\int f(1+|\nabla U|) d \mu \leq \int|\nabla f| d \mu
$$

Next replacing $f$ by $|f|^{p}(1+|\nabla U|)^{m p-1}$, we have

$$
\int|f|^{p}(1+|\nabla U|)^{m p} d \mu \leq \int \mid \nabla\left(|f|^{p}(1+|\nabla U|)^{m p-1} \mid\right) d \mu
$$

We discuss the right hand side as follows

$$
\begin{aligned}
\int \mid \nabla\left(|f|^{p}(1+|\nabla U|)^{m p-1}\right) d \mu \leq & p \int|\nabla f||f|^{p-1}(1+|\nabla U|)^{m p-1} \mid d \mu \\
& +(m p-1) \int|f|^{p}(1+|\nabla U|)^{m p-2}\left|\nabla^{2} U\right| d \mu
\end{aligned}
$$


Applying Youngs inequality to the first term on the right hand side, with dual indices $\frac{1}{p}+$ $\frac{1}{q}=1$, we have

$$
\begin{aligned}
& p \int|\nabla f||f|^{p-1}(1+|\nabla U|)^{m p-1} d \mu \\
& \quad \leq \epsilon^{-p / q} q^{-p / q} \frac{1}{p} \int|\nabla f|^{p}(1+|\nabla U|)^{(m-1) p} d \mu+\varepsilon \int|f|^{p}(1+|\nabla U|)^{m p} d \mu
\end{aligned}
$$

For the second term on the right hand side of Eq. 3, if we assume that

$$
\left|\nabla^{2} U\right| \leq C \frac{(1+|\nabla U|)^{2}}{\eta(|\nabla U|)}
$$

with some function $1 \leq \eta(x) \rightarrow_{x \rightarrow \infty} \infty$, then for any $\varepsilon \in(0,1)$ there is a constant $C_{\frac{\varepsilon}{(m p-1)}} \in(0, \infty)$ such that

$$
\left|\nabla^{2} U\right| \leq \frac{\varepsilon}{(m p-1)}(1+|\nabla U|)^{2}+C_{\frac{\varepsilon}{(m p-1)}}
$$

and hence

$$
(m p-1) \int|f|^{p}(1+|\nabla U|)^{m p-2}\left|\nabla^{2} U\right| d \mu \leq \varepsilon \int|f|^{p}(1+|\nabla U|)^{m p} d \mu+C_{\frac{\varepsilon}{(m p-1)}} \int|f|^{p} d \mu
$$

Combining (2)-(6), we arrive at

$$
\begin{aligned}
& \int|f|^{p}(1+|\nabla U|)^{m p} d \mu \leq \\
& \epsilon^{-p / q} q^{-p / q} \frac{1}{p} \int|\nabla f|^{p}(1+|\nabla U|)^{(m-1) p} d \mu+\varepsilon \int|f|^{p}(1+|\nabla U|)^{m p} d \mu \\
& +\varepsilon \int|f|^{p}(1+|\nabla U|)^{m p} d \mu+(m p-1) C_{\frac{\varepsilon}{(m p-1)}}^{m} \int|f|^{p} d \mu
\end{aligned}
$$

Choosing $\varepsilon \in\left(0, \frac{1}{2}\right)$ we arrive at the following bound

$$
\int|f|^{p}(1+|\nabla U|)^{m p} d \mu \leq \tilde{C} \int|\nabla f|^{p}(1+|\nabla U|)^{(m-1) p} d \mu+\tilde{D} \int|f|^{p} d \mu
$$

with some constant $\tilde{C} \equiv \tilde{C}(\varepsilon, p) \in(0, \infty)$ and $\tilde{D} \equiv \tilde{D}(\varepsilon, m, p) \in(0, \infty)$. From here we proceed by induction to conclude with the following bound

$$
\int|f|^{p}(1+|\nabla U|)^{m p} d \mu \leq C \sum_{k=1, . ., m} \int\left|\nabla^{k} f\right|^{p} d \mu+D \int|f|^{p} d \mu
$$

In an earlier paper [33], besides other interesting results, the following case was considered. Suppose $r>0$ be such that

$$
|U|^{r} \leq a\left(\frac{1}{4}|\nabla U|^{2}-\frac{1}{2} \Delta U+b\right)
$$

with some $a, b \in(0, \infty)$. Then for $m=1$ and $p=2$ the inequality holds with $A(s)=|s|^{r}$.

In both cases, [1] and [33], the results did not generalise to the infinite dimensional setting.

As an illustration of the results, in [1] a major class of examples was provided in the form of $U=U(d)$ where $d(x) \equiv d(x, 0)$ was a smooth function which coincided with the Euclidean distance function outside a ball centered at the origin.

Since the function $A(t)$ satisfies doubling condition, one can show the following bounded perturbation lemma, which includes non-regular $U$ 's, without much of a problem. 
Theorem 2 Suppose there exists a constant $K \in(0, \infty)$ such that for all $f \in W_{m, p}(\mu)$,

$$
\mu\left(\Phi_{A, p}(f)\right) \leq K\left(\|f\|_{m, p, \mu}^{p}+\Phi_{A, p}\left(\|f\|_{p, \mu}\right)\right) .
$$

Suppose

$$
d \tilde{\mu} \equiv e^{-V} d \mu
$$

with a bounded measurable function $V$. Then there is a constant $\tilde{K} \in(0, \infty)$ such that for all $f \in W_{m, p}(\tilde{\mu})$,

$$
\tilde{\mu}\left(\Phi_{A, p}(f)\right) \leq \tilde{K}\left(\|f\|_{m, p, \tilde{\mu}}^{p}+\Phi_{A, p}\left(\|f\|_{p, \tilde{\mu}}\right)\right) .
$$

where on the right hand side the norms are with the measure $\tilde{\mu}$.

We remark that one has the following equivalence of norms.

Theorem 3 Under the conditions of Lemma 1, suppose $U$ is locally bounded and $|\nabla U(x)| \rightarrow|x| \rightarrow \infty \infty$, then there exists a constant $C \in(0, \infty)$ such that

$$
\|f\|_{m, p}^{p} \leq C \mu\left(\left|\nabla^{m} f\right|^{p}+f^{p}\right)
$$

for any $f \in W_{m, p}(\mu)$. Thus the norms $\|f\|_{m, p}$ and

$$
\|f\|_{k, p}^{\sim} \equiv\|f\|_{p}+\left\|\nabla^{k} f\right\|_{p}
$$

are equivalent.

Proof Let $B \equiv B(0, R)$ be a ball of radius $R$ centered at the origin. We note a property that for Lebesgue measure $\lambda$, for any $1 \leq j<m$, one has [2]

$$
\int_{B}\left|\nabla^{j} f\right|^{p} d \lambda \leq c_{B}\left(\int_{B}\left|\nabla^{m} f\right|^{p}+|f|^{p} d \lambda\right) .
$$

with some constant $c_{B}$ independent of a function $f$. Then using local boundedness of $U$, with $\chi_{B}$ denoting the characteristic function of $B$, we get

$$
\begin{aligned}
& \mu\left(\left|\nabla^{j} f\right|^{p} \chi_{B}\right) \leq e^{-\inf _{B} U} \int_{B}\left|\nabla^{j} f\right|^{p} d \lambda
\end{aligned}
$$

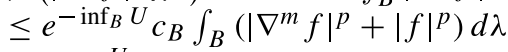

$$
\begin{aligned}
& \leq e^{o s c_{B} U_{c}} c_{B} \mu\left(\left|\nabla^{m} f\right|^{p}+|f|^{p}\right)
\end{aligned}
$$

where $\operatorname{osc}_{B} U \equiv \sup _{B}(U)-\inf _{B}(U)$. On the other hand using Lemma 1 and our assumption that $|\nabla U(x)| \rightarrow|x| \rightarrow \infty \infty$, we have

$$
\eta_{R} \equiv 1+\inf _{B^{c}}|\nabla U| \rightarrow_{R \rightarrow \infty} \infty
$$

and hence

$$
\begin{aligned}
& \mu\left(\left|\nabla^{j} f\right|^{p} \chi_{B^{c}}\right) \leq \frac{1}{\eta_{R}^{m-j}} \int_{B^{c}}\left|\nabla^{j} f\right|^{p}(1+|\nabla U|)^{(m-j) p} d \mu \\
& \leq \frac{1}{\eta_{R}^{m-j}} \mu\left(\left|\nabla^{j} f\right|^{p}(1+|\nabla U|)^{(m-j) p}\right) d \mu \\
& \leq \frac{{ }_{K}}{\eta_{R}^{m-j}}\|f\|_{m, p}^{p}
\end{aligned}
$$

Combining bounds (11)-(12), we get

$$
\mu\left(\left|\nabla^{j} f\right|^{p}\right) \leq e^{o s c_{B} U} c_{B} \mu\left(\left|\nabla^{m} f\right|^{p}+|f|^{p}\right)+\frac{K}{\eta_{R}^{m-j}}\|f\|_{m, p}^{p}
$$


Hence

$$
\begin{aligned}
\sum_{1 \leq j \leq m-1} \mu\left(\left|\nabla^{j} f\right|^{p}\right) \leq & {\left[(m-1) e^{o s c_{B} U} c_{B}+\frac{K}{\eta_{R}^{m-j}}\right] \mu\left(\left|\nabla^{m} f\right|^{p}+|f|^{p}\right) } \\
& +\sum_{1 \leq j \leq m-1} \frac{K}{\eta_{R}^{m-j}} \mu\left(\left|\nabla^{j} f\right|^{p}\right) .
\end{aligned}
$$

Taking $R \in(0, \infty)$ sufficiently large so that

$$
\frac{K}{\eta_{R}}<1
$$

this implies the desired property that there exists a constant $C \in(0, \infty)$ such that for all sufficiently large $R \in(0, \infty)$, we have

$$
\|f\|_{m, p}^{p} \leq C \mu\left(\left|\nabla^{m} f\right|^{p}+f^{p}\right)
$$

for any $f \in W_{m, p}(\mu)$.

Remark 1 We remark that other interesting applications of local perturbation technique can be found in the literature, see e.g. [3, 18, 23, 24],..., and others.

\section{Poincare's Inequalities of Higher Order}

We will show that under the conditions of Theorem 3, we also have a family of Poincaré inequalities satisfied.

Later on we denote by $\mathcal{P}_{k}$ the set of all polynomials on $\mathbb{R}^{n}$ of order $k$.

Lemma 2 Let $q \in(1, \infty], u \in L_{q}(\mu)$. Then there exists a unique $w \in \mathcal{P}_{k-1}$ such that

$$
\|u-w\|_{q}=\inf \left\{\|u-\alpha\|_{q}: \alpha \in \mathcal{P}_{k-1}\right\}
$$

Proof Let $d(u, q)=\inf \left\{\|u-\alpha\|_{q}: \alpha \in \mathcal{P}_{k-1}\right\}$. Firstly, we claim that if such the minimizer $w$ exists, we must have $\|w\|_{q} \leq 2\|u\|_{q}$. Otherwise,

$$
\|u-w\|_{q} \geq\|w\|_{q}-\|u\|_{q}>2\|u\|_{q}-\|u\|_{q}=\|u\|_{q}
$$

a contradiction to the definition of $w$. Hence we have

$$
d(u, q)=\inf \left\{\|u-\alpha\|_{q}:\|\alpha\|_{q} \leq 2\|u\|_{q} \text { and } \alpha \in \mathcal{P}_{k-1}\right\}
$$

Let $\mathcal{P}_{k-1}^{u}=\left\{\alpha \in \mathcal{P}_{k-1} \mid\|\alpha\|_{q} \leq 2\|u\|_{q}\right\}$. Recall that in our setup $1<q<\infty, L_{q}$ is reflexive. If $\left\{\alpha_{i}\right\} \subset \mathcal{P}_{k-1}^{u}$ is such that $\left\|u-\alpha_{i}\right\|_{q} \rightarrow d$, then there exists $w \in L_{q}$ so that $\alpha_{i} \rightarrow w$ in $L_{q}$. Since $\mathcal{P}_{k-1}^{u}$ is closed and convex, it is weakly closed. Thus $w \in \mathcal{P}_{k-1}^{u}$. Also notice that

$$
\|u-w\| \leq \liminf \left\|u-\alpha_{i}\right\|=d(u, q)
$$

We obtain the existence. 
Next, if $u$ itself is a polynomial, then the uniqueness is trivial. Otherwise, suppose there are $w_{1}$ and $w_{2}$ satisfying (13), then so is $\lambda w_{1}+(1-\lambda) w_{2}$. But

$$
\begin{aligned}
d(u, q) & =\left\|u-\lambda w_{1}+(1-\lambda) w_{2}\right\| \\
& =\left\|\lambda\left(u-w_{1}\right)+(1-\lambda)\left(u-w_{2}\right)\right\| \\
& \leq \lambda\left\|u-w_{1}\right\|+(1-\lambda)\left\|u-w_{2}\right\| \\
& =d(u, q)
\end{aligned}
$$

Since $L_{q}(\mu)$ is strictly convex, either $u=w_{1}$, or $u-w_{1}=\mu\left(u-w_{2}\right)$ for some $\mu$. Both of these cases indicate that $u$ itself is a polynomial, a contradiction.

For $q=\infty$, if $u$ is continuous, this is the Chebyshev Equioscillation Theorem. Otherwise, we can use density argument.

Definition 1 Given an integer $k, q \in(1, \infty]$ and $u \in L_{q}(\mu)$, the polynomial in Lemma 2 is called the $L_{q}$ minimizing polynomial of $u$ of order $k-1$, and is denoted as $M_{k, q}(u) \equiv$ $M_{k, q, \mu}(u)$.

In the framework of $L_{p}$ spaces with Lebsegue measure in balls the following inequalitites were established many years ago, see e.g. [37]. Let

$$
\left|\nabla^{k} f\right|^{p} \equiv \sum_{|\alpha|=k}\left|\nabla^{\alpha} f\right|^{p} .
$$

Let $B \equiv B(0, r) \subset \mathbb{R}^{n}$ be an open ball with radius $r$ centred at the origin. There exists $a$ constant $\tilde{c}_{k, p}(B) \equiv \tilde{c}_{k, p}(B, \lambda) \in(0, \infty)$ such that

$$
\int_{B}\left|f-M_{k, p, \lambda}(f)\right|^{p} d \lambda \leq \tilde{c}_{k, p}(B) \int\left|\nabla^{k} f\right|^{p} d \lambda
$$

With the coercive inequalities we established previously, we can obtain the higher order Poincare's inequalities for other probability measures.

Theorem 4 Suppose $1<q<\infty$ and $k \in \mathbb{N}$. Let $d \mu=e^{-U} d \lambda$ be such that $U$ satisfies Adams regularity condition, is locally bounded and $|\nabla U(x)| \rightarrow|x| \rightarrow \infty \infty$. Then there exists $c_{k, q} \in(0, \infty)$ such that

$$
\mu\left|f-M_{k, q, \mu}(f)\right|^{q} \leq c_{k, q} \mu\left|\nabla^{k} f\right|^{q}
$$

Proof We expand on the idea of [23]. First we note that with $w \equiv M_{k, q, \lambda, B}(f)$, defined with normalised Lebesgue measure in a ball $B_{r} \equiv B(0, r)$, we have

$$
\begin{aligned}
\int\left|f-M_{m, q, \mu}(f)\right|^{q} d \mu & \leq \int|f-w|^{q} d \mu \\
& \leq \int_{\left\{B_{r}\right\}}|f-w|^{q} d \mu+\int_{\left\{B_{r}^{c}\right\}}|f-w|^{q} d \mu
\end{aligned}
$$

For the first part, we have

$$
\begin{aligned}
\int_{\left\{B_{r}\right\}}|f-w|^{q} d \mu & \leq e^{\inf _{B_{r}}(U)} \int_{B_{R}}|f-w|^{q} d \lambda \\
& \leq e^{\inf _{B_{r}}(U)} \tilde{c}_{k, p}(B) \int_{B_{R}}\left|\nabla^{k} f\right|^{q} d \lambda \\
& \leq e^{o s c_{B_{r}}(U)} \tilde{c}_{k, p}(B) \int\left|\nabla^{k} f\right|^{q} d \mu
\end{aligned}
$$

For the second part, we note that with

$$
L \equiv \inf _{B_{r}^{c}}(1+|\nabla U|)
$$


by Lemma 1 and Theorem 3, we have

$$
\begin{aligned}
& \int_{B_{r}^{c}}|f-w|^{q} d \mu \\
\leq & \frac{1}{L} \int_{B_{r}^{c}}|f-w|^{q} \eta d \mu \\
\leq & \frac{1}{L} \int_{B_{r}^{c}}|f-w|^{q}(1+|\nabla U|)^{k q} \\
\leq & \frac{K}{L} \int\left|\nabla^{k} f\right|^{q} d \mu+\frac{K}{L} \int_{B_{r}^{c}}|f-w|^{q} d \mu
\end{aligned}
$$

Choosing $r \in(0, \infty)$ sufficiently large so that $L>K$, we obtain

$$
\int_{B_{r}^{c}}|f-w|^{q} d \mu \leq \frac{K}{(L-K)} \int\left|\nabla^{k} f\right|^{q} d \mu
$$

Combining (20)-(21), we get

$$
\int|f-w|^{q} d \mu \leq C \int\left|\nabla^{k} f\right|^{q} d \mu
$$

with

$$
C \equiv \max \left\{e^{o s c_{B_{r}}(U)} \tilde{c}_{k, p}(B), \frac{K}{(L-K)}\right\}
$$

Downhill Induction $\mathrm{L}_{q}$-Case Here we provide constructive arguments to demonstrate that in fact in our setup $\left(P I_{1, q}\right)$ implies the following inequalities of higher order.

Theorem 5 For $q \in(1, \infty)$, there exists $C_{k, q} \in(0, \infty)$ such that

$$
\mu\left|f-m_{k, q}(f)\right|^{q} \leq C_{k, q} \sum_{|\alpha|=k} \mu\left|\nabla^{\alpha} f\right|^{q},
$$

with some polynomial $m_{k, q}(f)$ of order $k-1$.

Proof Assume that $\left(P I_{1, q}\right)$ holds. Then

$$
\begin{aligned}
& \sum_{|\alpha|=k} \mu\left|\nabla^{\alpha} f\right|^{q}=\sum_{|\beta|=k-1} \sum_{j} \mu\left|\nabla_{j} \nabla^{\beta} f\right|^{q} \geq C_{1, q}^{-1} \sum_{|\beta|=k-1} \mu\left|\nabla^{\beta} f-M_{1, q}\left(\nabla^{\beta} f\right)\right|^{q} \\
= & C_{1, q}^{-1} \sum_{|\beta|=k-1} \mu\left|\nabla^{\beta}\left(f-B_{1, q, k-1}(f)\right)\right|^{q}
\end{aligned}
$$

with

$$
B_{1, q, k-1}(f) \equiv \sum_{|\beta|=k-1} \frac{x^{\beta}}{\beta !} M_{1, q}\left(\nabla^{\beta} f\right)
$$

By similar arguments applied inductively to the right hand side we obtain

$$
\sum_{|\alpha|=k} \mu\left|\nabla^{\alpha} f\right|^{q} \geq C_{1, q}^{-(k-j)} \sum_{|\alpha|=k-j} \mu\left|\nabla^{\alpha}\left(f-B_{1, q, j-1}(f)\right)\right|^{q}
$$

with

$$
B_{1, q, j-1}(f) \equiv \sum_{|\alpha|=k-j} \frac{x^{\alpha}}{\alpha !} M_{1, q}\left(\nabla^{\alpha}\left(f-B_{1, q, j}(f)\right)\right)
$$


This implies the desired result with the polynomial $m_{k, q} \equiv B_{1, q, 1}(f)$ and a constant $C_{k, q} \leq$ $C_{1, q}^{k}$.

The constant in the above result grows exponentially in $k$ and may be not optimal, and different then the optimal constant in the $\left(P I_{k, q}\right)$ with the minimizing polynomial $M_{k, q}(f)$. At the current stage of knowledge the optimal constants is still a delicate issue. We only know how to determine them for the $\mathrm{O}-\mathrm{U}$ case.

\section{Orlicz-Sobolev Inequalities}

\section{Shift type minimizers for Orlicz norms.}

Let $\Phi$ be an Orlicz function and let

$$
\|f\|_{\Phi} \equiv \inf \left\{\lambda>0: \mu\left(\Phi\left(\frac{f}{\lambda}\right)\right) \leq 1\right\}
$$

be the corresponding Luxemburg norm.

Theorem 6 Suppose the norm $\|\cdot\|_{\Phi}$ is strictly convex. Then there exists unique functional $M_{\Phi, \mu}(f)$ such that

$$
\left\|f-M_{\Phi, \mu}(f)\right\|_{\Phi} \equiv \inf _{a \in \mathbb{R}}\|f-a\|_{\Phi}
$$

Moreover we have

$$
M_{\Phi, \mu}(s f)=|s| M_{\Phi, \mu}(f)
$$

and

$$
\left\|(s f+t g)-M_{\Phi, \mu}(s f+t g)\right\|_{\Phi} \leq|s|\left\|f-M_{\Phi, \mu}(f)\right\|_{\Phi}+|t|\left\|g-M_{\Phi, \mu}(g)\right\|_{\Phi}
$$

Let $\Phi$ be an Orlicz function. Then we have

$$
\left|\mu(f)-M_{\Phi, \mu}(f)\right| \leq \Phi^{-1}(1)\left\|f-M_{\Phi, \mu}(f)\right\|_{\Phi} \leq \Phi^{-1}(1)\|f-\mu(f)\|_{\Phi}
$$

and

$$
\|f-\mu(f)\|_{\Phi} \leq\left(1+\Phi^{-1}(1) \cdot\|\mathbb{I}\|_{\Phi}\right)\left\|f-M_{\Phi, \mu}(f)\right\|_{\Phi}
$$

We have the following continuity property.

$$
\left|\left\|f-M_{\Phi, \mu}(f)\right\|_{\Phi}-\left\|g-M_{\Phi, \mu}(g)\right\|_{\Phi}\right| \leq\|g-f\|_{\Phi} .
$$

Remark 2 By definition of $M_{\Phi, \mu}(f)$, we have

$$
\left\|f-M_{\Phi, \mu}(f)\right\|_{\Phi} \leq\|f-\mu(f)\|_{\Phi}
$$

Proof Using the definition of infimum and Minkowski inequality, we have

$$
\begin{aligned}
& \left\|(s f+t g)-M_{\Phi, \mu}(s f+t g)\right\|_{\Phi} \leq\left\|(s f+t g)-\left(s M_{\Phi, \mu}(f)+t M_{\Phi, \mu}(g)\right)\right\|_{\Phi} \\
\leq & |s|\left\|f-M_{\Phi, \mu}(f)\right\|_{\Phi}+|t|\left\|g-M_{\Phi, \mu}(g)\right\|_{\Phi}
\end{aligned}
$$

First inequality in the second part follows via Jensen inequality from the convexity of $\Phi$ using the definition of the Luxemburg norm. The right hand side inequality follows from 
the definition of the minimizer $M_{\Phi, \mu}(f)$. The proof of second statement is as follows, using Minkowski inequality together with the inequality proven above, we have

$$
\begin{aligned}
& \|f-\mu(f)\|_{\Phi} \leq\left|\mu(f)-M_{\Phi, \mu}(f)\right| \cdot\|\mathbb{I}\|_{\Phi}+\left\|f-M_{\Phi, \mu}(f)\right\|_{\Phi} \\
\leq & \left(1+\Phi^{-1}(1) \cdot\|\mathbb{I}\|_{\Phi}\right)\left\|f-M_{\Phi, \mu}(f)\right\|_{\Phi} .
\end{aligned}
$$

To show the last statement, note that by definition

$$
\left\|f-M_{\Phi, \mu}(f)\right\|_{\Phi}=\inf _{a \in \mathbb{R}}\|f-a\|_{\Phi} \leq\|f-g\|_{\Phi}+\left\|g-M_{\Phi, \mu}(g)\right\|_{\Phi}
$$

and similarly

$$
\left\|g-M_{\Phi, \mu}(g)\right\|_{\Phi} \leq\|g-f\|_{\Phi}+\left\|f-M_{\Phi, \mu}(f)\right\|_{\Phi} .
$$

Hence

$$
\left|\left\|f-M_{\Phi, \mu}(f)\right\|_{\Phi}-\left\|g-M_{\Phi, \mu}(g)\right\|_{\Phi}\right| \leq\|g-f\|_{\Phi} .
$$

\section{Perturbation of measures and norms.}

In this paragraph we will discuss the change of objects introduced before when the measure changes. Therefore we adopt now a notation which explicitly shows dependence of the norm on the measure.

Theorem 7 Consider a probability measure

$$
d v=e^{-V} d \mu,
$$

defined with $\|V\|_{\infty}<\infty$. Then we have

$$
e^{\inf (V)}\|f\|_{\Phi, v} \leq\|f\|_{\Phi, \mu} \leq e^{\sup (V)}\|f\|_{\Phi, v}
$$

Proof : For such a measure $v$, we have

$$
1=v\left(\Phi\left(\frac{f}{\|f\|_{\Phi, v}}\right)\right) \geq e^{-\sup (U)} \mu\left(\Phi\left(\frac{f}{\|f\|_{\Phi, v}}\right)\right) \geq \mu\left(\Phi\left(\frac{f}{e^{\sup (U)}\|f\|_{\Phi, v}}\right)\right)
$$

Hence, using the definition of Luxemburg norm we get

$$
\|f\|_{\Phi, \mu} \leq e^{\sup (U)}\|f\|_{\Phi, v}
$$

Similarly

$$
1=\mu\left(\Phi\left(\frac{f}{\|f\|_{\Phi, \mu}}\right)\right) \geq e^{\inf (U)} v\left(\Phi\left(\frac{f}{\|f\|_{\Phi, \mu}}\right)\right)
$$

and since $\int e^{U} d v=1$, we have $\inf U<0$, which by convexity of $\Phi$ yields

$$
1 \geq v\left(\Phi\left(\frac{f}{e^{-\inf (U)}\|f\|_{\Phi, \mu}}\right)\right) .
$$

From that we conclude that

$$
e^{-\inf (U)}\|f\|_{\Phi, \mu} \geq\|f\|_{\Phi, \nu}
$$


Condition for Minimum Point Suppose $\Phi \in \Delta_{2}$, i.e. satisfies the doubling condition $\Phi(2 x) \leq C \Phi(x)$ with some constant $C \in(0, \infty)$ for all $x \in \mathbb{R}$. Then we have

$$
\mu \Phi\left(\frac{f}{\|f\|_{\Phi}}\right)=1
$$

This implies that

$$
\frac{d}{d a} \mu \Phi\left(\frac{f-a}{\|f-a\|_{\Phi}}\right)=0
$$

which implies the following condition for an extremal point

$$
\frac{d}{d a}\|f-a\|_{\Phi}=\frac{\mu\left(\Phi^{\prime}\left(\frac{f-a}{\|f-a\|_{\Phi}}\right)\right)}{\mu\left(\Phi^{\prime}\left(\frac{f-a}{\|f-a\|_{\Phi}}\right) \frac{f-a}{\|f-a\|_{\Phi}^{2}}\right)}
$$

Hence we get the following necessary condition for the minimal point $M_{\Phi}(f)$.

\section{Proposition 1}

$$
\mu\left(\Phi^{\prime}\left(\frac{f-M_{\Phi}(f)}{\left\|f-M_{\Phi}(f)\right\|_{\Phi}}\right)\right)=0
$$

Example: Special case of $L_{p}$ norms For $p>2$ using convexity we have

$$
\left\|f-M_{p, \mu}(f)\right\|_{p}^{2} \leq\left(\mu(f)-M_{p, \mu}(f)\right)^{2}+(p-1)\|f-\mu(f)\|_{p}^{2} .
$$

For $p \in[1,2)$, we have

$$
\left\|f-M_{p, \mu}(f)\right\|_{p}^{2} \geq\left(\mu(f)-M_{p, \mu}(f)\right)^{2}+(p-1)\|f-\mu(f)\|_{p}^{2}
$$

and hence

$$
\|f-\mu(f)\|_{p} \leq(p-1)^{-\frac{1}{2}}\left\|f-M_{p, \mu}(f)\right\|_{p} .
$$

In general, if $\mu$ is a probability measure, we have

$$
\left|\mu(f)-M_{p, \mu}(f)\right| \leq\left\|f-M_{p, \mu}(f)\right\|_{1} \leq\left\|f-M_{p, \mu}(f)\right\|_{p} \leq\|f-\mu(f)\|_{p} .
$$

Using this we have

$$
\|f-\mu(f)\|_{p} \leq\left\|f-M_{p, \mu}(f)\right\|_{p}+\left|\mu(f)-M_{p, \mu}(f)\right| \leq 2\left\|f-M_{p, \mu}(f)\right\|_{p}
$$

Hence, we get the following property.

Proposition 2 For any $p \in[1, \infty)$ and a probability measure $\mu$, we have

$$
\frac{1}{2}\|f-\mu(f)\|_{p} \leq \sup _{z \in \mathbb{R}}\left\|(f+z)-M_{p, \mu}(f+z)\right\|_{p} \leq\|f-\mu(f)\|_{p}
$$

and

$$
\sup _{z \in \mathbb{R}}\left|\left(M_{p, \mu}(f+z)-z\right)\right| \leq|\mu f|+\|f-\mu(f)\|_{p}
$$

Later we will consider an increasing family of closed linear subspaces $\mathcal{K}_{n} \subset \mathcal{K}_{n+1}$ and will be interested in corresponding minimizers.

$$
\left\|f-M_{\Phi, n, \mu}(f)\right\|_{\Phi} \equiv \inf _{\alpha \in \mathcal{K}_{n}}\|f-\alpha\|_{\Phi}
$$


Since by our assumption $\mathcal{K}_{n}$ is a linear space, for any $h \in \mathcal{K}_{n}$ we have

$$
\begin{array}{r}
\left\|f+h-M_{\Phi, n, \mu}(f+h)\right\|_{\Phi} \equiv \inf _{\alpha \in \mathcal{K}_{n}}\|f+h-\alpha\|_{\Phi} \\
=\inf _{\alpha \in \mathcal{K}_{n}}\|f-(\alpha-h)\|_{\Phi}=\inf _{\alpha \in \mathcal{K}_{n}}\|f-\alpha\|_{\Phi}
\end{array}
$$

Hence we get the following result.

Proposition 3 For any $h \in \mathcal{K}_{n}$, we have

$$
\left\|f+h-M_{\Phi, n, \mu}(f+h)\right\|_{\Phi}=\left\|f-M_{\Phi, n, \mu}(f)\right\|_{\Phi}
$$

and

$$
\left\|M_{\Phi, n, \mu}(f+h)-h\right\|_{\Phi} \leq\|f\|_{\Phi}+\left\|f-M_{\Phi, n, \mu}(f)\right\|_{\Phi}
$$

Remark In particular if $M_{\Phi, n, \mu}(f)$ is unique, we have

$$
\forall h \in \mathcal{K}_{n} \quad M_{\Phi, n, \mu}(f+h)-h=M_{\Phi, n, \mu}(f)
$$

Definition 2 We say that a probability measure $\mu$ satisfies $(\Phi-\Psi)$ Orlicz-Sobolev Inequality iff $\exists C \in(0, \infty)$ such that the following relation holds

$$
\left\|f-M_{\Phi, \mu}(f)\right\|_{\Phi, \mu} \leq C\||\nabla f|\|_{\Psi, \mu}
$$

for any $f$ for which the right hand side is finite.

Remark: Naturally such inequalities are of more interest if the norm $\|\cdot\| \Psi, \mu$ is weaker than $\|\cdot\|_{\Phi, \mu}$

Theorem 8 (Perturbation Lemma) Suppose

$$
d \nu=e^{-V} d \mu
$$

is a probability measure defined with $\|V\|_{\infty}<\infty$. If $\mu$ satisfies $(\Phi-\Psi)$ Orlicz-Sobolev Inequality with a constant $C_{\mu} \in(0, \infty)$, then also it is satisfied by $v$ with a constant not larger than

$$
C_{v}=e^{o s c(V)} C_{\mu}
$$

Proof Using definition of the minimiser and comparison of Orlicz norms theorem, we have

$$
\left\|f-M_{\Phi, v}(f)\right\|_{\Phi, v} \leq\left\|f-M_{\Phi, \mu}(f)\right\|_{\Phi, v} \leq e^{-\inf (U)}\left\|f-M_{\Phi, \mu}(f)\right\|_{\Phi, \mu}
$$

Next using $(\Phi-\Psi)$ Orlicz-Poincare Inequality for the measure $\mu$ together with comparison of Orlicz norms lemma, we have

$$
e^{-\inf (U)}\left\|f-M_{\Phi, \mu}(f)\right\|_{\Phi, \mu} \leq e^{-\inf (U)} C\||\nabla f|\| \Psi, \mu \leq e^{\sup (U)-\inf (U)} C\||\nabla f|\|_{\Psi, \nu}
$$

Combining both relations yields the desired result.

\section{Example: Log-Sobolev inequality}

A probability measure $\mu$ satisfies Log-Sobolev inequality with respect to a sub-gradient $\nabla \equiv\left(X_{1}, . ., X_{k}\right)$ iff $\exists c \in(0, \infty)$

$$
E_{n} t_{\mu}\left(f^{2}\right) \equiv \mu\left(f^{2} \log \frac{f^{2}}{\mu\left(f^{2}\right)}\right) \leq c \mu|\nabla f|^{2}
$$


for all functions $f$ for which the right hand side is well defined.

It was observed in [14] [BG] that, since the right hand side is invariant with respect to the shift by a constant, the left hand side can be replaced by a functional

$$
\mathfrak{L}(f) \equiv \sup _{a \in \mathbb{R}} \operatorname{Ent}_{\mu}\left((f-a)^{2}\right)
$$

with sup well defined (thanks to Rothaus lemma) and the Log-Sobolev inequality is equivalent to the following relation

$$
\|f-\mu f\|_{N} \leq \tilde{c}\||\nabla f|\|_{\mathbb{L}_{2}}
$$

with an Orlicz function

$$
N(x) \equiv x^{2} \log \left(1+x^{2}\right) .
$$

According the theory developed above this inequality is equivalent to

$$
\left\|f-M_{N, \mu} f\right\|_{N} \leq \hat{c}\||\nabla f|\|_{\mathbb{L}_{2}},
$$

with a constant $\hat{c} \in(0, \infty)$ independent of $f$, and this inequality admits simple bounded perturbation theory of the probability measures.

\section{Orlicz-Sobolev Inequalities of Higher Order Let}

$$
\mathcal{H}_{k} \equiv\left\{g \in \mathbb{L}_{\Phi}(\mu): \nabla^{k} g=0\right\}
$$

For $k \in \mathbb{N}$, define a functional of order $k$

$$
\left\|f-M_{k, \Phi, \mu}(f)\right\|_{\Phi} \equiv \inf _{g \in \mathcal{H}_{k}}\|f-g\|_{\Phi, \mu}
$$

Recall for the case $k=1$, we could use $\mathbb{L}_{2}$ minimizer to dominate the minimum. For $k>1$ the choice of $\mathbb{L}_{2}$ minimizer may be not possible and depends both on $\Phi$ and $\mu$. This makes the analysis interesting and more flexible. In this context one can study the following Orlicz-Poincaré inequalities of higher order

$$
\left\|f-M_{k, \Phi, \mu}(f)\right\|_{\Phi} \leq c_{k, \Phi} \mu\left|\nabla^{k} f\right|^{2} .
$$

\section{Tight Orlicz-Sobolev Inequalities of Higher Order}

In this section we introduce and study a tight analog of Adams bounds in the form of the following inequalities.

Definition 3 We say that a probability measure $v$ satisfies $(\Phi-\Psi)$ Orlicz-Sobolev Inequality of order $k \in \mathbb{N}$ iff $\exists C_{k} \in(0, \infty)$ such that the following relation holds

$$
\left\|f-M_{k, \Phi, \mu}(f)\right\|_{\Phi, \mu} \leq C_{k}\left\|\left|\nabla^{k} f\right|\right\|_{\Psi, \mu}
$$

for any $f$ for which the right hand side is finite.

For $j \in \mathbb{N}$, we define $\mathrm{j}$-th composition of exponential function $\exp _{j+1}(t):=$ $\exp \left(\exp _{j}(t)\right)$, with $\exp _{1}(t) \equiv e^{t}$. Then for $t \geq e_{j} \equiv \exp _{j}(1)$, we set $\log _{j+1}(t):=$ $\log \left(\log _{j} t\right)$, with a convention that $\log _{1} \equiv \log$. Similarly letting $\log ^{*}(t)=\max \{1, \log (t)\}$, we define its $j$-th composition $\log _{j}^{*}(t)$. 
Lemma 3 For any $j \in \mathbb{N}$ and $p \geq 1$, we have for any $x \in \mathbb{R}$ the following relation

$$
\frac{1}{C_{j}} \log _{j}\left(\gamma_{j}+|x|^{p}\right) \leq \log _{j}\left(\gamma_{j}+|x|\right)
$$

with $C_{1}=p$ and $\gamma_{1}=1$, whereas for $j \geq 2$,

with $C_{j}=2$ and $\gamma_{j+1} \equiv \max \left\{e_{j}, p, \gamma_{j}\right\}$.

Proof We prove the lemma by induction. When $j=1$, taking $\gamma_{1}=1$, we have the following elementary inequality $(a+b)^{p} \geq a+b^{p}$ for $b>0$ and $a \geq 1$. With $a=\gamma \geq \gamma_{1} \equiv 1$ and $b=|x|$, we get

$$
p \log (\gamma+|x|) \geq \log \left(\gamma+|x|^{p}\right)
$$

i.e. we obtain the desired estimate with $C_{1}=p$. Hence, applying log to both sides above, for $\gamma \geq \max \{e, p\}$, we get

$$
2 \log _{2}(\gamma+|x|) \geq \log p+\log _{2}(\gamma+|x|) \geq \log _{2}\left(\gamma+|x|^{p}\right)
$$

Next suppose for $j \geq 2$ we have

$$
2 \log _{j}(\gamma+|x|) \geq \log _{j}\left(\gamma+|x|^{p}\right)
$$

for all $\gamma \geq \gamma_{j} \equiv \max \left\{e_{j-1}, p, \gamma_{j-1}\right\}$. For $\gamma \geq \gamma_{j+1} \equiv \max \left\{e_{j}, p, \gamma_{j}\right\}$, taking log on both sides, we have

$$
2 \log _{j+1}(\gamma+|x|) \geq \log 2+\log _{j+1}(\gamma+|x|) \geq \log _{j+1}\left(\gamma+|x|^{p}\right) .
$$

Lemma 4 For all $j \in \mathbb{N}$, and all $|x| \geq e_{j-1}$,

$$
\log _{j}(e|x|) \leq 2 \log _{j}^{*}(|x|)
$$

Proof If $j=1$, for all $|x|>0$ we have

$$
\log (e|x|)=1+\log |x| \leq 2 \log ^{*}(|x|)
$$

Suppose for a given $j \in \mathbb{N}$ and all $|x| \geq e_{j-1}$, we have

$$
\log _{j}(e|x|) \leq 2 \log _{j}^{*}(|x|)
$$

For $|x| \geq e_{j}$, taking log of both sides of the above, we arrive at

$$
\begin{aligned}
\log _{j+1}(e|x|) & =\log \left(\log _{j}(e|x|)\right) \leq \log \left(2 \log _{j}^{*}(|x|)\right) \\
& \leq \log 2+\log _{j+1}^{*}(|x|) \leq 2 \log _{j+1}^{*}(|x|)
\end{aligned}
$$

Lemma $5 \exists D_{j} \in(0, \infty)$ s.t.

$$
\log _{j}\left(\gamma_{j}+|x|\right) \leq D_{j} \log _{j}^{*}(|x|)
$$

for all $x \in \mathbb{R}$.

Proof When $|x| \geq \gamma_{j}$, by Lemma 4, we have

$$
\log _{j}\left(\gamma_{j}+|x|\right) \leq \log _{j}(2|x|) \leq 2 \log _{j}^{*}(|x|)
$$

For $|x| \leq \gamma_{j}$, the function

$$
\frac{\log _{j}\left(\gamma_{j}+|x|\right)}{\log _{j}^{*}(|x|)}
$$


is continuous function in $\left[-\gamma_{j}, \gamma_{j}\right]$. Hence it can be bounded by some constant $D_{j}$.

Corollary 1 Let

$$
\Phi(t)=t \prod_{j=1}^{n}\left(\log _{j}\left(\gamma_{j}+|t|\right)\right)^{p_{j}} \equiv t \boldsymbol{\Theta}(t)
$$

and

$$
\Phi_{A, p}(t)=|t|^{p} \prod_{j=1}^{n}\left(\log _{j}^{*}(|t|)\right)^{p_{j}} \equiv|t|^{p} A\left(\log ^{*} t\right) .
$$

Then there exists $C>0$ such that

$$
\Phi\left(|x|^{p}\right) \leq C \Phi_{A, p}(|x|) .
$$

Proof Using Lemmas 3-5 above,

$$
\begin{aligned}
\Phi\left(|x|^{p}\right) & =|x|^{p} \prod_{j=1}^{n}\left(\log _{j}\left(\gamma_{j}+|x|^{p}\right)\right)^{p_{j}} \\
& \leq|x|^{p} \prod_{j=1}^{n}\left(C_{j} D_{j} \log _{j}^{*}(|t|)\right)^{p_{j}} \\
& =C|x|^{p} \prod_{j=1}^{n}\left(\log _{j}^{*}(|x|)\right)^{p_{j}}
\end{aligned}
$$

where $C=\prod_{j=1}^{n} C_{j} D_{j}$.

\section{Lemma 6}

$$
\|f\|_{p}^{p} \leq \Phi^{-1}(1)\left\|f^{p}\right\|_{\Phi}
$$

Proof By Jensen's Inequality,

$$
1=\int \Phi\left(\frac{|f|^{p}}{\left\|f^{p}\right\|_{\Phi}}\right) d \mu \geq \Phi\left(\int \frac{|f|^{p}}{\left\|f^{p}\right\|_{\Phi}} d \mu\right)
$$

Applying monotone function $\Phi^{-1}$ to both sides yields

$$
\|f\|_{p}^{p} \leq \Phi^{-1}(1)\left\|f^{p}\right\|_{\Phi} .
$$

Theorem 9 Suppose the following Adams Inequality holds

$$
\mu\left(\Phi_{A, p}(f)\right) \leq \tilde{C}_{A} \mu\left|\nabla^{k} f\right|^{p}+\tilde{D}_{A} \Phi_{A, p}\left(\|f\|_{p}^{p}\right)
$$

with some $\tilde{C}_{A}, \tilde{D}_{A} \in(0, \infty)$ independent of $f$. Then

$$
\left\||f|^{p}\right\|_{\Phi} \leq C^{\prime} \mu\left|\nabla^{k} f\right|^{p}+D^{\prime}\|f\|_{p}^{p} .
$$

with some $C^{\prime}, D^{\prime} \in(0, \infty)$ independent of $f$. Moreover if the following $(p, k)$-Poincaré inequality holds

$$
\left\|\left(f-M_{p, k}(f)\right)\right\|_{p}^{p} \leq c_{p, k} \mu\left|\nabla^{k} f\right|^{p}
$$


with some $c_{p, k} \in(0, \infty)$ for all $f$ for which the right hand side is well defined, then the following tight $(\Phi, p, k)$-Inequality holds

$$
\left\|\left|f-M_{p, k}(f)\right|^{p}\right\|_{\Phi} \leq C \mu\left|\nabla^{k} f\right|^{p} .
$$

Conversely (OSI) implies ( $\Phi, p, k)$-Inequality with a constant

$$
c_{p, k}=C \Phi^{-1}(1)
$$

Proof By Lemmas 3-5 the (AI) implies the following inequality

$$
\mu\left(\Phi\left(|f|^{p}\right)\right) \leq C^{\prime} \mu\left|\nabla^{k} f\right|^{p}+D^{\prime} \Phi_{A, p}\left(\|f\|_{p}^{p}\right) .
$$

with some constants $C^{\prime}, D^{\prime} \in(0, \infty)$ independent of the function $f$ for which the right hand side is well defined. Applying this inequality with a function $f /\left\||f|^{p}\right\|_{\Phi}^{\frac{1}{p}}$ for which the right hands side is equal to one, we obtain

$$
\begin{aligned}
1= & \mu\left(\Phi\left(\left|f /\left\||f|^{p}\right\|_{\Phi}^{\frac{1}{p}}\right|^{p}\right)\right) \leq C^{\prime} \mu\left|\nabla^{k} f /\left\||f|^{p}\right\|_{\Phi}^{\frac{1}{p}}\right|^{p} \\
& +D^{\prime} \mu\left(\left|f /\left\||f|^{p}\right\|_{\Phi}^{\frac{1}{p}}\right|^{p}\right) A\left(\mu\left(\left|f /\left\||f|^{p}\right\|_{\Phi}^{\frac{1}{p}}\right|^{p}\right)\right) \\
\leq & C^{\prime} \mu\left|\nabla^{k} f /\left\||f|^{p}\right\|_{\Phi}^{\frac{1}{p}}\right|^{p}+D^{\prime} A\left(\Phi^{-1}(1)\right) \mu|f|^{p} /\left\||f|^{p}\right\|_{\Phi}
\end{aligned}
$$

and hence using Lemma 6 we arrive at (AOI). Applying (AOI) to a function $f-M_{p, k}(f)$ and using the ( $\left.\mathrm{PI}_{p, k}\right)$, we conclude with the tight inequality (OSI).

On the other hand, using (OSI) together with Lemma 6 we get $\left(\mathrm{PI}_{p, k}\right)$ with the desired constant.

Remark 3 Recall that in a particular case, see [15] and [35], if AOI holds for $p=2$ with the Dirichlet form of a Pearson generator, then the generator has a discrete spectrum. Thus Poincaré inequality and, by our down hill induction, Poincaré inequality of higher order hold.

\section{Relation of the Norms}

Let $d \mu=e^{-U} d \lambda$, where $U$ is a smooth function in $\mathbb{R}^{n}$. The adjoint of the gradient in the corresponding Hilbert space is given by $\nabla^{*}=-\nabla+\nabla U$. Consider the Friedrichs extension of the following Dirichlet operator

$$
L \equiv \nabla^{*} \cdot \nabla=-\sum_{j=1, . ., n} \nabla_{j}^{2}+\sum_{j=1, . ., n} \nabla_{j}(U) \nabla_{j} \equiv-\Delta+\nabla U \cdot \nabla
$$

defined initially on the dense set of smooth compactly supported functions. Then the extension, denoted later on by the same symbol $L$, is a positive, self-adjoint operator for which $L^{\frac{1}{2}}$ is a well-defined, positive and self-adjoint linear operator.

In this section we study equivalence of norms defined in terms of higher order derivatives and powers of the operator $L$, respectively given as follows with $k \in \mathbb{N}$ and $p \in[1, \infty)$.

$$
\|f\|_{L, k, p} \equiv\|f\|_{p}+\left\|L^{\frac{k}{2}} f\right\|_{p}
$$


and

$$
\|f\|_{k, p}^{\sim} \equiv\|f\|_{p}+\left\|\nabla^{k} f\right\|_{p}
$$

which is equivalent under our assumptions to $\|f\|_{k, p}$ via Theorem 3 . We begin from discussing Hilbert space norms corresponding to $p=2$. Our first task is to show that using the Adams regularity condition on the function $U$, we can show the desired equivalence for $k \leq 3$. This will also serve as an useful introduction of ideas and techniques which will be pushed up later when discussing the general case. In the general case we will require additional regularity assumption on the log of the density function.

Theorem 10 Let $d \mu=e^{-U} d \lambda$, with a Adams regular function $U$, for which $|\nabla U| \rightarrow|x| \rightarrow \infty$ $\infty$. Then the norms $\|f\|_{L, k, 2},\|f\|_{k, 2}$ and $\|f\|_{k, 2}^{\sim}$ defined on $W_{k, 2}, k \leq 3$ are equivalent.

Proof Because of Theorem 3, it is sufficient to show equivalence of $\|f\|_{L, k, 2}$ and $\|f\|_{k, 2}^{\sim}$. For $k=1$ we have equality of norms, because

$$
\left\|L^{\frac{1}{2}} f\right\|_{2}^{2}=\mu(f L f)=\|\nabla f\|_{2} .
$$

For $k=2$ we have

$$
\begin{aligned}
\|L f\|_{2}^{2}= & \mu\left(f L^{2} f\right)=\sum_{i, j} \mu\left(\nabla_{i} f\left(\nabla_{i} \nabla_{j}^{*}\right) \nabla_{j} f\right) \\
= & \sum_{i, j} \mu\left(\nabla_{i} f\left(-\nabla_{i} \nabla_{j}+\nabla_{i} \nabla_{j}(U)\right) \nabla_{j} f\right) \\
= & \sum_{i, j} \mu\left(\nabla_{i} f\left(-\nabla_{j}+\nabla_{j}(U) \nabla_{i}-\nabla_{j}(U) \nabla_{i}+\nabla_{i} \nabla_{j}(U)\right) \nabla_{j} f\right) \\
= & \sum_{i, j} \mu\left(\left|\nabla_{j} \nabla_{i} f\right|^{2}\right) \\
& -\sum_{i, j} \mu\left(\nabla_{j}(U)\left(\nabla_{i} f\right) \nabla_{i} \nabla_{j}(f)\right) \\
& +\sum_{i, j} \mu\left(\left(\nabla_{i} \nabla_{j}(U)\right) \nabla_{i}(f) \nabla_{j}(f)\right)
\end{aligned}
$$

By Cauchy-Schwartz inequality and application of Lemma 1 and Theorem 3, we get

$$
\begin{array}{r}
\left|\sum_{i, j} \mu\left(\nabla_{j}(U)\left(\nabla_{i} f\right) \nabla_{i} \nabla_{j}(f)\right)\right| \leq \frac{1}{2} \sum_{i, j} \mu\left|\nabla_{i} \nabla_{j}(f)\right|^{2}+\frac{1}{2} \mu\left(|\nabla f|^{2}(1+|\nabla U|)^{2}\right) \\
\leq \tilde{C}_{1}\|f\|_{2,2}^{2}
\end{array}
$$

with some constant $\tilde{C}_{1} \in(0, \infty)$ independent of $f$. By similar arguments based on CauchySchwartz inequality and application of Lemma 1 and Theorem 3 , with some constant $\tilde{C}_{2} \in$ $(0, \infty)$, we get

$$
\left|\sum_{i, j} \mu\left(\left(\nabla_{i} \nabla_{j}(U)\right) \nabla_{i}(f) \nabla_{j}(f)\right)\right| \leq \mu\left(|\nabla f|^{2} \cdot(1+|\nabla U|)^{2-\varepsilon}\right) \leq \tilde{C}_{2}\|f\|_{2,2}^{2}
$$

Hence

$$
\|f\|_{L, 1,2}^{2} \leq C_{2}\|f\|_{2,2}^{2}
$$


with some constant $0<C_{2} \leq 2(C+1)$. For $k=3 / 2$, we have

$$
\begin{aligned}
\left\|L^{\frac{3}{2}} f\right\|_{2}^{2} & =\mu\left(|\nabla(L f)|^{2}\right)=\mu\left(|L \nabla(f)+[\nabla, L](f)|^{2}\right) \\
& =\sum_{i} \mu\left(\left|L \nabla_{i}(f)+\left[\nabla_{i}, L\right](f)\right|^{2}\right) \\
& \leq 2 \sum_{i} \mu\left(\left|L \nabla_{i}(f)\right|^{2}\right)+2 \sum_{i} \mu\left(\left|\left[\nabla_{i}, L\right](f)\right|^{2}\right)
\end{aligned}
$$

where in the last step we have used the operator convexity of the quadratic function. The first on the right hand side can be bounded using the case $k=1$. For the second term, with some constants $C^{\prime}, C \in(0, \infty)$, we have

$$
\begin{aligned}
& \sum_{i} \mu\left(\left|\left[\nabla_{i}, L\right](f)\right|^{2}=\sum_{i} \mu\left(\left|\sum_{j} \nabla_{i} \nabla_{j}(U) \cdot \nabla_{j}(f)\right|^{2}\right.\right. \\
& \leq \mu\left(|\nabla(f)|^{2}(1+|\nabla u|)^{2-\varepsilon}\right) \leq C^{\prime}\|f\|_{2,2}^{2} \leq C\|f\|_{3,2}^{2}
\end{aligned}
$$

Combining all the above we arrive at

$$
\|f\|_{L, 3,2}^{2} \leq C_{3}\|f\|_{3,2}^{2}
$$

with some constant $C_{3} \in(0, \infty)$ independent of $f$.

Conversely, we have

$$
\begin{aligned}
\mu\left(\left|\nabla^{2} f\right|^{2}\right) & \equiv \sum_{i, j} \mu\left(\left|\nabla_{i} \nabla_{j} f\right|^{2}\right)=\sum_{i, j} \mu\left(\nabla_{i} f \cdot \nabla_{j}^{*} \nabla_{i} \nabla_{j} f\right) \\
& =\sum_{i, j} \mu\left(\nabla_{i}^{*} \nabla_{i}(f) \cdot \nabla_{j}^{*} \nabla_{j} f\right)+\sum_{i, j} \mu\left(\nabla_{i}(f) \cdot\left[\nabla_{j}^{*}, \nabla_{i}\right] \nabla_{j} f\right) \\
& =\|f\|_{L, 1,2}^{2}-\sum_{i, j} \mu\left(\nabla_{i}(f) \cdot \nabla_{j} \nabla_{i}(U) \cdot \nabla_{j}(f)\right) \\
& \leq\|f\|_{L, 1,2}^{2}+C \mu\left(|\nabla(f)|^{2}(1+|\nabla U|)^{2-\varepsilon}\right) \\
& \leq\|f\|_{L, 1,2}^{2}+C\left(\mu\left(|\nabla(f)|^{2}\right)\right)^{\varepsilon}\left(\mu\left(|\nabla(f)|^{2}(1+|\nabla U|)^{2}\right)\right)^{\frac{2-\varepsilon}{2}} \\
& \leq\|f\|_{L, 1,2}^{2}+\varepsilon C^{1 / \varepsilon} K^{\frac{2-\varepsilon}{2}} \mu\left(|\nabla(f)|^{2}\right)+\frac{2-\varepsilon}{2}\|f\|_{2,2}^{2}
\end{aligned}
$$

Hence

$$
\begin{aligned}
\mu\left(\left|\nabla^{2} f\right|^{2}\right) & \leq \frac{2}{\varepsilon}\|f\|_{L, 1,2}^{2}+2 C^{1 / \varepsilon} K^{\frac{2-\varepsilon}{2}} \mu\left(|\nabla(f)|^{2}\right)+\frac{2-\varepsilon}{\varepsilon}\|f\|_{2}^{2} \\
& \leq\left(\frac{2}{\varepsilon}+C^{1 / \varepsilon} K^{\frac{2-\varepsilon}{2}}\right)\|f\|_{L, 1,2}^{2}+\frac{2-\varepsilon}{\varepsilon}\|f\|_{2}^{2} \\
& \leq\left(C^{1 / \varepsilon} K^{\frac{2-\varepsilon}{2}}+\frac{4-\varepsilon}{\varepsilon}\right)\|f\|_{L, 1,2}^{2}
\end{aligned}
$$


For the higher norm

$$
\begin{aligned}
\mu\left(\left|\nabla^{3} f\right|^{2}\right) & \equiv \sum_{i, j, k} \mu\left(\left|\nabla_{i} \nabla_{j} \nabla_{k} f\right|^{2}\right)=\sum_{i, j, k} \mu\left(\nabla_{i} \nabla_{j} f \cdot \nabla_{k}^{*} \nabla_{k} \nabla_{i} \nabla_{j} f\right) \\
& =\sum_{i, j, k} \mu\left(\nabla_{i}^{*} \nabla_{i} \nabla_{j}(f) \cdot \nabla_{k}^{*} \nabla_{k} \nabla_{j} f\right)+\sum_{i, j, k} \mu\left(\nabla_{i} \nabla_{j}(f) \cdot\left[\nabla_{k}^{*}, \nabla_{i}\right] \nabla_{j} \nabla_{k} f\right) \\
& \leq \mu(L \nabla(f) \cdot L \nabla(f))++\mu\left(\sum_{i, k}\left|\nabla_{k} \nabla_{i}(U)\right|\left|\nabla^{2} f\right|^{2}\right) \\
& \leq \mu(L \nabla(f) \cdot L \nabla(f))+C \mu\left(\left|\nabla^{2} f\right|^{2}(1+|\nabla U|)^{2-\varepsilon}\right) \\
& \leq \mu(L \nabla(f) \cdot L \nabla(f))+C\left(\mu\left(\left|\nabla^{2}(f)\right|^{2}\right)\right)^{\varepsilon}\left(\mu\left(\left|\nabla^{2}(f)\right|^{2}(1+|\nabla U|)^{2}\right)\right)^{\frac{2-\varepsilon}{2}} \\
& \leq \mu(L \nabla(f) \cdot L \nabla f)+\varepsilon C^{1 / \varepsilon} K^{\frac{2-\varepsilon}{2}} \mu\left(\left|\nabla^{2}(f)\right|^{2}\right)+\frac{2-\varepsilon}{2}\|f\|_{3,2}^{2}
\end{aligned}
$$

Hence we obtain

$$
\mu\left(\left|\nabla^{3} f\right|^{2}\right) \leq \frac{2}{\varepsilon} \mu(L \nabla(f) \cdot L \nabla f)+2 C^{1 / \varepsilon} K^{\frac{2-\varepsilon}{2}} \mu\left(\left|\nabla^{2}(f)\right|^{2}\right)+\frac{2-\varepsilon}{\varepsilon}\|f\|_{2}^{2}
$$

For the first term on the right hand side, we have

$$
\begin{aligned}
& \mu(L \nabla(f) \cdot L \nabla f)=\mu\left(f L^{3}(f)\right)+2 \sum_{j} \mu\left(\left[L, \nabla_{j}\right](f) \cdot \nabla_{j} L f\right) \\
& \quad+\sum_{j} \mu\left(\left[L, \nabla_{j}\right](f) \cdot\left[L, \nabla_{j}\right](f)\right)
\end{aligned}
$$

The middle one in Eq. 39 can be treated as follows

$$
\begin{aligned}
\sum_{j} \mu\left(\left[L, \nabla_{j}\right](f) \cdot \nabla_{j} L f\right) & =-\sum_{j, k} \mu\left(\nabla_{j} \nabla_{k}(U) \nabla_{k}(f) \cdot \nabla_{j} L f\right) \\
& \leq \mu\left(\sum_{j, k}\left|\nabla_{j} \nabla_{k}(U)\right| \cdot|\nabla f| \cdot|\nabla L f|\right) \\
& \leq \mu\left((1+|\nabla U|)^{2-\varepsilon} \cdot|\nabla f| \cdot|\nabla L f|\right) \\
& \frac{1}{2} \mu\left(f L^{3} f\right)+C^{2} \mu\left(|\nabla f|^{2}(1+|\nabla U|)^{(2-\varepsilon) 2}\right) \\
& \leq \frac{1}{2} \mu\left(f L^{3} f\right)+C^{2}\left(\mu\left(|\nabla f|^{2}\right)\right)^{\varepsilon}\left(\mu\left(|\nabla f|^{2}(1+|\nabla U|)^{2 \cdot 2}\right)\right)^{\frac{2-\varepsilon}{2}} \\
& \leq \frac{1}{2} \mu\left(f L^{3} f\right)+C^{2}\left(\mu\left(|\nabla f|^{2}\right)\right)^{\varepsilon}\left(D\|f\|_{3,2}^{2}\right)^{\frac{2-\varepsilon}{2}} \\
& \leq \frac{1}{2} \mu\left(f L^{3} f\right)+\varepsilon C^{2 / \varepsilon}(2 D)^{\frac{2-\varepsilon}{2}}\left(\mu|\nabla f|^{2}\right)+\frac{1}{2} \frac{2-\varepsilon}{2}\|f\|_{3,2}^{2}
\end{aligned}
$$


with some $C, D \in(0, \infty)$ independent of $f$. Finally the last one in Eq. 39 can be treated as follows

$$
\begin{aligned}
\sum_{j} \mu\left(\left[L, \nabla_{j}\right](f) \cdot\left[L, \nabla_{j}\right](f)\right) & =\sum_{j} \mu\left(\sum_{k, l} \nabla_{k} \nabla_{j}(U) \nabla_{l} \nabla_{j}(U) \cdot \nabla_{k} f \nabla_{l} f\right) \\
\leq & \mu\left((1+|\nabla U|)^{(2-\varepsilon) 2}|\nabla f|^{2}\right) \\
\leq & \left(\mu|\nabla f|^{2}\right)^{\varepsilon}\left(\mu\left((1+|\nabla U|)^{4}|\nabla f|^{2}\right)\right)^{\frac{2-\varepsilon}{2}} \\
\leq & \left(\mu|\nabla f|^{2}\right)^{\varepsilon}\left(D\|f\|_{3,2}^{2}\right)^{\frac{2-\varepsilon}{2}} \\
& \varepsilon(D)^{\frac{2-\varepsilon}{2}} \mu|\nabla f|^{2}+\frac{2-\varepsilon}{2}\|f\|_{3,2}^{2}
\end{aligned}
$$

with some constant $D \in(0, \infty)$ independent of $f$. Combining the above inequalities we arrive at the desired bound

$$
\mu\left(\left|\nabla^{3} f\right|^{2}\right) \leq D^{\prime}\|f\|_{L, 3,2}^{2}
$$

with some constant $D^{\prime} \in(0, \infty)$ independent of $f$.

To consider the more general case we introduce the following assumption on derivatives of $U$ of higher order up to an order $3 \leq m \in \mathbb{N}$.

Assumption $\left(A_{m}\right)$ There exists a constant $K \in(0, \infty)$ such that

$$
\begin{aligned}
\exists K \in(0, \infty) & \left|\nabla^{2} U\right| \leq K(1+|\nabla U|)^{2-\epsilon} \\
\forall 3 \leq k \leq m \exists K_{k} \in(0, \infty) & \left|\nabla^{k} U\right| \leq K_{k}(1+|\nabla U|)^{k},
\end{aligned}
$$

Remark 4 The assumption $\left(\mathbf{A}_{m}\right)$ includes a vast family of functions $U$. In particular it includes semibounded polynomials and any $\Phi_{j}, j \in \mathbb{Z}^{+}$classes of [1] for which it holds for any $m \in \mathbb{N}$.

Theorem 11 Suppose the assumption $\left(\boldsymbol{A}_{\bar{m}}\right)$ is satisfied and $|\nabla U| \rightarrow_{|x| \rightarrow \infty} \infty$. Then for any $k \leq \bar{m}$ the norms $\|f\|_{L, k, 2},\|f\|_{k, 2}$ and $\|f\|_{k, 2}^{\sim}$ are equivalent.

Proof By Adam's Lemma 1, the condition $\left(\mathbf{A}_{\bar{m}}\right)$ implies

$$
\int\left|\nabla^{k} U\right|^{2} f^{2} d \mu \leq K\|f\|_{k, 2}^{2}
$$

When $m=1,2$, the proof has been done above, see Theorem 10 . Assume now it holds for all $m^{\prime}$ such that $3 \leq m^{\prime} \leq m-1$. By inductive assumption, Leibnitz rule and Eq. 41 , we have

$$
\begin{aligned}
\left\|L^{\frac{m}{2}} f\right\|_{2} & =\left\|L^{\frac{m-2}{2}}(L f)\right\|_{2} \\
& \leq C\left(\|L f\|_{2}+\left\|\nabla^{m-2}(L f)\right\|_{2}\right) \\
& \leq C_{1}\left(\|f\|_{2}+\left\|\nabla^{2} f\right\|_{2}\right)+\left\|\nabla^{m-2}\left(-\nabla^{2} f+\nabla U \nabla f\right)\right\|_{2} \\
& \leq C_{1}\left(\|f\|_{2}+\left\|\nabla^{2} f\right\|_{2}\right)+\left\|\nabla^{m} f\right\|_{2}+\left\|\nabla^{m-2}(\nabla U \nabla f)\right\|_{2}
\end{aligned}
$$


with some constants $C, C_{1} \in(0, \infty)$ independent of $f$. For the last term on the right hand side we have

$$
\begin{aligned}
\left\|\nabla^{m-2}(\nabla U \nabla f)\right\|_{2}^{2} \equiv & \sum_{j_{1}, \ldots, j_{m-2}}\left\|\nabla_{j_{1}, . ., j_{m-2}}^{m-2}\left(\sum_{l} \nabla_{l} U \nabla_{l} f\right)\right\|_{2}^{2} \\
& =\sum_{j_{1}, . ., j_{m-2}}\left\|\sum_{l}\left(\left[\nabla_{j_{1}, . ., j_{m-2}}^{m-2}, \nabla_{l} U\right] \nabla_{l} f+\nabla_{l} U \nabla_{j_{1}, \ldots, j_{m-2}}^{m-2} \nabla_{l} f\right)\right\|_{2}^{2} \\
& \leq 2 \sum_{j_{1}, \ldots, j_{m-2}}\left\|\sum_{l}\left(\left[\nabla_{j_{1}, \ldots, j_{m-2}}^{m-2}, \nabla_{l} U\right]\right) \nabla_{l} f\right\|_{2}^{2} \\
& +2 \sum_{j_{1}, \ldots, j_{m-2}}\left\|\sum_{l} \nabla_{l} U \nabla_{j_{1}, \ldots, j_{m-2}}^{m-2} \nabla_{l} f\right\|_{2}^{2} .
\end{aligned}
$$

For the last term on the right hand side of Eq. 43 we use Adams' lemma to get

$$
2 \sum_{j_{1}, . ., j_{m-2}}\left\|\sum_{l} \nabla_{l} U \nabla_{j_{1}, . ., j_{m-2}}^{m-2} \nabla_{l} f\right\|_{2}^{2} \leq C_{3}\|f\|_{m, 2}^{2}
$$

with some constant $C_{3} \in(0, \infty)$ independent of $f$. For the first term on the right hand side of Eq. 43 we use the following formula for the commutator which can be proven inductively.

\section{Lemma 7}

$$
\left[\nabla_{j_{1}, \ldots, j_{m-2}}^{m-2}, F\right]=\sum_{s=1}^{m-2} \sum_{\mathbf{j}_{s}, \mathbf{i}_{m-2-s}}\left(\nabla_{\mathbf{j}_{s}}^{s} F\right) \nabla_{\mathbf{i}_{m-2-s}}^{m-2-s}
$$

where $\mathbf{j}_{s} \equiv\left\{j_{r_{1}}, . ., j_{r_{s}}\right\}$ and $\mathbf{i}_{m-2-s}\left\{j_{r_{s+1}}, . ., j_{r_{m-2}}\right\}$ are partitions of $j_{1}, . ., j_{m-2}$, of cardinality $s$ and $m-2-s$, respectively.

Applying this lemma with $F=\nabla_{l} U$, we have

$$
\left[\nabla_{j_{1}, . ., j_{m-2}}^{m-2}, \nabla_{l} U\right]=\sum_{s=1}^{m-2} \sum_{\mathbf{j}_{s}, \mathbf{i}_{m-2-s}}\left(\nabla_{\mathbf{j}_{s}}^{s} \nabla_{l} U\right) \nabla_{\mathbf{i}_{m-2-s}}^{m-2-s}
$$

with corresponding partitions $\mathbf{j}_{s} \equiv\left\{j_{r_{1}}, . ., j_{r_{s}}\right\}$ and $\mathbf{i}_{m-2-s}\left\{j_{r_{s+1}}, . ., j_{r_{m-2}}\right\}$ of $j_{1}, . ., j_{m-2}$, of cardinality $s$ and $m-2-s$, respectively. We get

$$
\begin{aligned}
& 2 \sum_{j_{1}, \ldots, j_{m-2}}\left\|\sum_{l}\left(\left[\nabla_{j_{1}, . ., j_{m-2}}^{m-2}, \nabla_{l} U\right]\right) \nabla_{l} f\right\|_{2}^{2} \\
& =2 \sum_{j_{1}, . ., j_{m-2}}\left\|\sum_{l} \sum_{s=1}^{m-2} \sum_{\mathbf{j}_{s}, \mathbf{i}_{m-2-s}}\left(\nabla_{\mathbf{j}_{s}}^{s} \nabla_{l} U\right) \nabla_{\mathbf{i}_{m-2-s}}^{m-2-s} \nabla_{l} f\right\|_{2}^{2} \\
& \leq 2(m-2) \sum_{s=1} \sum_{s=1}^{m-2} \sum_{j_{1}, . ., j_{m-2}}\left\|\sum_{l} \sum_{\mathbf{j}_{s}, \mathbf{i}_{m-2-s}}\left(\nabla_{\mathbf{j}_{s}}^{s} \nabla_{l} U\right) \nabla_{\mathbf{i}_{m-2-s}}^{m-2-s} \nabla_{l} f\right\|_{2}^{2} \\
& \leq 2(m-2) \sum_{s=1}^{m-2} \sum_{s=1}^{m-2} \sum_{j_{1}, . ., j_{m-2}} \sum_{l, l^{\prime}} \sum_{\mathbf{j}_{s}, \mathbf{i}_{m-2-s}, \mathbf{j}_{s}^{\prime}, \mathbf{i}_{m-2-s}^{\prime}} \\
& \int\left(\left(\nabla_{\mathbf{j}_{s}}^{s} \nabla_{l} U\right) \nabla_{\mathbf{i}_{m-2-s}}^{m-2-s} \nabla_{l} f\right)\left(\left(\nabla_{\mathbf{j}_{s}^{\prime}}^{s} \nabla_{l} U\right) \nabla_{\mathbf{i}_{m-2-s}^{\prime}}^{m-2-s} \nabla_{l^{\prime}} f\right) d \mu
\end{aligned}
$$


Hence

$$
\begin{aligned}
& 2 \sum_{j_{1}, ., j_{m-2}}\left\|\sum_{l}\left(\left[\nabla_{j_{1}, . ., j_{m-2}}^{m-2}, \nabla_{l} U\right]\right) \nabla_{l} f\right\|_{2}^{2} \\
& \leq 2(m-2) \sum_{s=1}^{m-2} \sum_{s=1}^{m-2} \sum_{j_{1}, . ., j_{m-2}} \sum_{l, l^{\prime}} \sum_{\mathbf{j}_{s}, \mathbf{i}_{m-2-s}, \mathbf{j}_{s}^{\prime}, \mathbf{i}_{m-2-s}^{\prime}} \\
& \left(\int\left|\left(\nabla_{\mathbf{j}_{s}}^{s} \nabla_{l} U\right) \nabla_{\mathbf{i}_{m-2-s}}^{m-2-s} \nabla_{l} f\right|^{2} d \mu\right)^{\frac{1}{2}}\left(\int\left|\left(\nabla_{\mathbf{j}_{s}^{\prime}}^{s} \nabla_{l} U\right) \nabla_{\mathbf{i}_{m-2-s}^{\prime}}^{m-2-s} \nabla_{l^{\prime}} f\right|^{2} d \mu\right)^{\frac{1}{2}}
\end{aligned}
$$

Since by our assumption about the derivatives of $U$ in the condition $\left(\mathbf{A}_{\bar{m}}\right)$ and Adams' Lemma 1, we have

$$
\begin{gathered}
\int\left|\left(\nabla_{\mathbf{j}_{s}}^{s} \nabla_{l} U\right) \nabla_{\mathbf{i}_{m-2-s}}^{m-2-s} \nabla_{l} f\right|^{2} d \mu \leq K\left\|\nabla_{\mathbf{i}_{m-2-s}}^{m-2-s} \nabla_{l} f\right\|_{s+1}^{2} \\
\int\left|\left(\nabla_{\mathbf{j}_{s}^{\prime}}^{s} \nabla_{l} U\right) \nabla_{\mathbf{i}_{m-2-s}^{\prime}}^{m-2-s} \nabla_{l^{\prime}} f\right|^{2} d \mu \leq K\left\|\nabla_{\mathbf{i}_{m-2-s}^{\prime}}^{m-2-s} \nabla_{l^{\prime}} f\right\|_{s+1}^{2}
\end{gathered}
$$

we conclude that with some constant $C^{\prime} \in(0, \infty)$, we have

$$
2 \sum_{j_{1}, . ., j_{m-2}}\left\|\sum_{l}\left(\left[\nabla_{j_{1}, . ., j_{m-2}}^{m-2}, \nabla_{l} U\right]\right) \nabla_{l} f\right\|_{2}^{2} \leq C^{\prime}\|f\|_{m, 2}^{2}
$$

Combining (43)-(50) and using Theorem 3 we conclude that

$$
\left\|L^{\frac{m}{2}} f\right\|_{2} \leq C ”\|f\|_{m, 2}
$$

with some constant $C$ " $\in(0, \infty)$ independent of $f$. This ends the proof of the inductive step and implies the first part of the theorem.

In order to proceed further we will need to have a control on constants in the Adam's bounds as follows.

Theorem 12 Let $m \geq 2$ be an integer. Suppose the assumption $\left(\boldsymbol{A}_{m}\right)$ holds. Then $\exists \gamma>0$, $\forall p \in[2-\gamma, \infty), \forall \epsilon>0, \exists K>0, \forall f \in W_{k, p}(\mu)$,

$$
\int\left|\nabla^{k} U\right|^{p}|f|^{p} d \mu \leq \epsilon\left\|\nabla^{k} f\right\|_{p}^{p}+K\|f\|_{p}^{p}
$$

for all $k \leq m$.

The proof of this theorem is based on the following lemma.

Lemma 8 Under the assumption $\left(\boldsymbol{A}_{m}\right)$, we have

$\exists \gamma>0, \forall p \in[2-\gamma, \infty), \exists K>0, \forall f \in W_{m, p}(\mu)$,

$$
\int(1+|\nabla U|)^{2^{m-1} p}|f|^{p} d \mu \leq K\|f\|_{m, p}^{p}
$$

where $\|f\|_{m, p} \equiv\|f\|_{m, p, \mu}$ is the weighted Sobolev norm in the space $W_{m, p}(\mu)$.

Proof of Lemma 8 Arguing as at the beginning of the proof of Theorem 1 for $U \equiv U(d)$, we have

$$
\int f(1+|\nabla U|) d \mu \leq \int|\nabla f| d \mu
$$


Replacing $f$ with $|f|^{p}(1+|\nabla U|)^{2^{m-1} p-1}$, we have

$$
\begin{aligned}
\int|f|^{p}(1+|\nabla U|)^{2^{m-1} p} d \mu & \leq \int \mid \nabla\left(|f|^{p}(1+|\nabla U|)^{2^{m-1} p-1}\right) d \mu \\
& \leq p \int|\nabla f||f|^{p-1}(1+|\nabla U|)^{2^{m-1} p-1} d \mu \\
& +\left(2^{m-1} p-1\right) \int|f|^{p}(1+|\nabla U|)^{2^{m-1}} p-2\left|\nabla^{2} U\right| d \mu
\end{aligned}
$$

For the first term on the right hand side of Eq. 54, under the assumption $p \geq 2-2^{2-m}$, $\forall \epsilon>0, \exists C(\epsilon)>0$ such that

$$
\begin{aligned}
& \int|\nabla f||f|^{p-1}(1+|\nabla U|)^{2^{m-1} p-1} d \mu \\
\leq & \int|f|^{p-1}(1+|\nabla U|)^{2^{m-1}(p-1)} \cdot|\nabla f|(1+|\nabla U|)^{2^{m-2} p} d \mu \\
\leq & \epsilon \int|f|^{p}(1+|\nabla U|)^{2^{m-1}} p d \mu+C(\epsilon) \int|\nabla f|^{p}(1+|\nabla U|)^{2^{m-2}} d \mu
\end{aligned}
$$

For the second term on the right hand side of Eq. 54

$$
\begin{aligned}
\int|f|^{p}(1+|\nabla U|)^{2^{m-1} p-2}\left|\nabla^{2} U\right| d \mu & \leq C \int|f|^{p}(1+|\nabla U|)^{2^{m-1} p-\delta} d \mu \\
& \leq \epsilon \int|f|^{p}(1+|\nabla U|)^{2^{m-1} p} d \mu+C(\epsilon) \int|f|^{p} d \mu
\end{aligned}
$$

Taking $\epsilon=\frac{1}{4}$ in both estimates above, we arrive at

$$
\frac{1}{2} \int|f|^{p}(1+|\nabla U|)^{2^{m-1} p} d \mu \leq K\left(\int|\nabla f|^{p}(1+|\nabla U|)^{2^{m-2} p} d \mu+\int|f|^{p} d \mu\right)
$$

From here we proceed by induction to conclude with the following bound

$$
\int(1+|\nabla U|)^{2^{m-1} p}|f|^{p} d \mu \leq K\|f\|_{m, p, \mu}^{p}
$$

In fact, if $p \geq 2-2^{2-m}$, then for all $j \leq m, p \geq 2-2^{2-j}$, hence

$$
\begin{aligned}
\int|f|^{p}(1+|\nabla U|)^{2^{m-1} p} d \mu \leq & K\left(\int|\nabla f|^{p}(1+|\nabla U|)^{2^{m-2} p} d \mu+\int|f|^{p} d \mu\right) \\
& \leq K^{2}\left(\int\left|\nabla^{2} f\right|^{p}(1+|\nabla U|)^{2^{m-3} p} d \mu+\int|f|^{p} d \mu+\int|\nabla f|^{p} d \mu\right) \\
\leq & \cdots \\
\leq & K^{m-2}\left(\int\left|\nabla^{m-2} f\right|^{p}(1+|\nabla U|)^{2 p} d \mu\right. \\
& \left.+\int|f|^{p} d \mu+\int|\nabla f|^{p} d \mu+\cdots+\int\left|\nabla^{m-3} f\right|^{p} d \mu\right) \\
\leq & K^{m-1}\left(\int\left|\nabla^{m-1} f\right|^{p}(1+|\nabla U|)^{p} d \mu\right. \\
& \left.+\int|f|^{p} d \mu+\int|\nabla f|^{p} d \mu+\cdots+\int\left|\nabla^{m-2} f\right|^{p} d \mu\right) \\
\leq & C\|f\|_{m, p, \mu}^{p}
\end{aligned}
$$

where the last line is due to the Adam's inequality of Lemma 1. 
Remark 5 From the proof we can see that the inequality holds when $p \in\left[2-2^{2-m}, \infty\right)$.

Assuming Lemma 8 we complete the proof of the Theorem 12 as follows.

Completion of the Proof of Theorem 12 For any $\epsilon>0$, we have

$$
\begin{aligned}
\int\left|\nabla^{m} U\right|^{p}|f|^{p} d \mu & \leq C \int(1+|\nabla U|)^{\left(2^{m-1}-\delta_{m}\right) p}|f|^{p} d \mu \\
& \leq \epsilon \int(1+|\nabla U|)^{2^{m-1} p}|f|^{p} d \mu+K(\epsilon) \int|f|^{p} d \mu \\
& \leq \epsilon\left(\left\|\nabla^{m} f\right\|_{p}^{p}+\|f\|_{p}^{p}\right)+K(\epsilon)\|f\|_{p}^{p} \\
& \leq \epsilon\left\|\nabla^{m} f\right\|_{p}^{p}+K\|f\|_{p}^{p}
\end{aligned}
$$

with some constants $K(\epsilon), K \in(0, \infty)$ independent of $f$. (Note that in the first inequality we have actually used a weaker condition than $\left(A_{m}\right)$ ).

Theorem 13 Let $d \mu=e^{-U} d \lambda$, with a function $U$ for which $|\nabla U|_{\rightarrow|x| \rightarrow \infty} \infty$ and which satisfies assumption $\left(\boldsymbol{A}_{m}\right)$, for $m \in \mathbb{N}$. Then the norms $\|f\|_{L, k, 2},\|f\|_{k, 2}$ and $\|f\|_{k, 2}^{\sim}$, are equivalent on $W_{k, 2}$, for $k \in \mathbb{N}, k \leq m$.

Proof The proof is via induction. The cases $k=1,2$, are included into Theorem 10. Suppose the statement holds for all $k$ such that $3 \leq k \leq m^{\prime}-1$. We will show that it also holds for $m^{\prime} \leq m$. Using the inductive assumption, with some constants $\bar{C}, C \in(0, \infty)$, we have

$$
\begin{aligned}
\left\|L^{\frac{m}{2}} f\right\|_{2} & =\left\|L^{\frac{m-2}{2}}(L f)\right\|_{2} \\
& \leq \bar{C}\left(\|L f\|_{2}+\left\|\nabla^{m-2}(L f)\right\|_{2}\right) \\
& \leq C\left(\|f\|_{2}+\left\|\nabla^{2} f\right\|_{2}\right)+\bar{C}\left\|\nabla^{m-2}\left(-\nabla^{2} f+\nabla U \nabla f\right)\right\|_{2} \\
& \leq C\left(\|f\|_{2}+\left\|\nabla^{2} f\right\|_{2}\right)+\bar{C}\left\|\nabla^{m} f\right\|_{2}+\bar{C}\left\|\nabla^{m-2}(\nabla U \nabla f)\right\|_{2}
\end{aligned}
$$

For the last term on the right hand side of this inequality, we have

$$
\begin{aligned}
\left\|\nabla^{m-2}(\nabla U \nabla f)\right\|_{2}^{2} & \equiv \sum_{j_{1}, \ldots, j_{m-2}}\left\|\nabla_{j_{1}, \ldots, j_{m-2}}^{m-2}\left(\sum_{l} \nabla_{l} U \nabla_{l} f\right)\right\|_{2}^{2} \\
& =\sum_{j_{1}, \ldots, j_{m-2}}\left\|\sum_{l}\left(\left[\nabla_{j_{1}, \ldots, j_{m-2}}^{m-2}, \nabla_{l} U\right] \nabla_{l} f+\nabla_{l} U \nabla_{j_{1}, \ldots, j_{m-2}}^{m-2} \nabla_{l} f\right)\right\|_{2}^{2} \\
& \leq \sum_{j_{1}, \ldots, j_{m-2}}\left\|\sum_{l}\left(\left[\nabla_{j_{1}, \ldots, j_{m-2}}^{m-2}, \nabla_{l} U\right]\right) \nabla_{l} f\right\|_{2}^{2} \\
& +2 \sum_{j_{1}, \ldots, j_{m-2}}\left\|\sum_{l} \nabla_{l} U \nabla_{j_{1}, \ldots, j_{m-2}}^{m-2} \nabla_{l} f\right\|_{2}^{2}
\end{aligned}
$$

For the last term on the right hand side, we use the Adams' inequality to get

$$
2 \sum_{j_{1}, \ldots, j_{m-2}}\left\|\sum_{l} \nabla_{l} U \nabla_{j_{1}, \ldots, j_{m-2}}^{m-2} \nabla_{l} f\right\|_{2}^{2} \leq \tilde{C}\|f\|_{m, 2}^{2}
$$


with some constant $\tilde{C} \in(0, \infty)$ independent of $f$. For the first term on the right hand side involving the commutator, we use the formula for the commutator of Lemma 7

$$
\left[\nabla_{j_{1}, \ldots, j_{m-2}}^{m-2}, \nabla_{l} U\right]=\sum_{s=1}^{m-2} \sum_{\mathbf{j}_{s}, \mathbf{i}_{m-2-s}}\left(\nabla_{\mathbf{j}_{s}}^{s} \nabla_{l} U\right) \nabla_{\mathbf{i}_{m-2-s}}^{m-2-s}
$$

with $\mathbf{j}_{s} \equiv\left\{j_{r_{1}}, \ldots, j_{r_{s}}\right\}$ and $\mathbf{i}_{m-2-s}\left\{j_{r_{s+1}}, \ldots, j_{r_{m-2}}\right\}$ are partitions of $j_{1}, \ldots, j_{m-2}$ of cardinality $s$ and $m-2-s$, respectively, to get

$$
\begin{aligned}
& 2 \sum_{j_{1}, \ldots, j_{m-2}}\left\|\sum_{l}\left(\left[\nabla_{j_{1}, \ldots, j_{m-2}}^{m-2}, \nabla_{l} U\right]\right) \nabla_{l} f\right\|_{2}^{2} \\
= & 2 \sum_{j_{1}, \ldots, j_{m-2}}\left\|\sum_{l} \sum_{s=1}^{m-2} \sum_{\mathbf{j}_{s}, \mathbf{i}_{m-2-s}}\left(\nabla_{\mathbf{j}_{s}}^{s} \nabla_{l} U\right) \nabla_{\mathbf{i}_{m-2-s}}^{m-2-s} \nabla_{l} f\right\|_{2}^{2} \\
\leq & 2(m-2) \sum_{s=1}^{m-2} \sum_{s=1}^{m-2} \sum_{j_{1}, \ldots, j_{m-2}}\left\|\sum_{l} \sum_{\mathbf{j}_{s}, \mathbf{i}_{m-2-s}}\left(\nabla_{\mathbf{j}_{s}}^{s} \nabla_{l} U\right) \nabla_{\mathbf{i}_{m-2-s}}^{m-2-s} \nabla_{l} f\right\|_{2}^{2} \\
\leq & 2(m-2) \sum_{s=1}^{m-2} \sum_{s=1}^{m-2} \sum_{j_{1}, \ldots, j_{m-2}} \sum_{l, l^{\prime} \mathbf{j}_{s}, \mathbf{i}_{m-2-s}, \mathbf{j}_{s}^{\prime}, \mathbf{i}_{m-2-s}^{\prime}} \sum_{\left(\left(\nabla_{\mathbf{j}_{s}}^{s} \nabla_{l} U\right) \nabla_{\mathbf{i}_{m-2-s}}^{m-2-s} \nabla_{l} f\right)}\left(\left(\nabla_{\mathbf{j}_{s}^{\prime}}^{s} \nabla_{l} U\right) \nabla_{\mathbf{i}_{m-2-s}^{\prime}-2-s}^{m} \nabla_{l^{\prime}} f\right) d \mu
\end{aligned}
$$

Hence

$$
\begin{aligned}
& \quad \sum_{j_{1}, \ldots, j_{m-2}}\left\|\sum_{l}\left(\left[\nabla_{j_{1}, \ldots, j_{m-2}}^{m-2}, \nabla_{l} U\right]\right) \nabla_{l} f\right\|_{2}^{2} \\
& \leq 2(m-2) \sum_{s=1}^{m-2} \sum_{s=1}^{m-2} \sum_{j_{1}, \ldots, j_{m-2}} \sum_{l, l^{\prime}} \sum_{\mathbf{j}_{s}, \mathbf{i}_{m-2-s}, \mathbf{j}_{s}^{\prime}, \mathbf{i}_{m-2-s}^{\prime}} \\
& \quad\left(\int\left|\left(\nabla_{\mathbf{j}_{s}}^{s} \nabla_{l} U\right) \nabla_{\mathbf{i}_{m-2-s}}^{m-2-s} \nabla_{l} f\right|^{2} d \mu\right)^{\frac{1}{2}}\left(\int\left|\left(\nabla_{\mathbf{j}_{s}^{\prime}}^{s} \nabla_{l} U\right) \nabla_{\mathbf{i}_{m-2-s}^{\prime}}^{m-2-s} \nabla_{l^{\prime}} f\right|^{2} d \mu\right)^{\frac{1}{2}}
\end{aligned}
$$

since by our assumption about the derivatives of $U$ and the revised Adams' inequality of Theorem 12, we have

$$
\begin{aligned}
& \int\left|\left(\nabla_{\mathbf{j}_{s}}^{s} \nabla_{l} U\right) \nabla_{\mathbf{i}_{m-2-s}}^{m-2-s} \nabla_{l} f\right|^{2} d \mu \leq K\left\|\nabla_{\mathbf{i}_{m-2-s}}^{m-2-s} \nabla_{l} f\right\|_{s+1}^{2} \\
& \int\left|\left(\nabla_{\mathbf{j}_{s}^{\prime}}^{s} \nabla_{l} U\right) \nabla_{\mathbf{i}_{m-2-s}^{\prime}}^{m-2-s} \nabla_{l^{\prime}} f\right|^{2} d \mu \leq K\left\|\nabla_{\mathbf{i}_{m-2-s}^{\prime}}^{m-2-s} \nabla_{l^{\prime}} f\right\|_{s+1}^{2}
\end{aligned}
$$

we conclude that with some constant $C^{\prime} \in(0, \infty)$, we have

$$
2 \sum_{j_{1}, \ldots, j_{m-2}}\left\|\sum_{l}\left(\left[\nabla_{j_{1}, \ldots, j_{m-2}}^{m-2}, \nabla_{l} U\right]\right) \nabla_{l} f\right\|_{2}^{2} \leq C^{\prime}\|f\|_{m, 2}^{2}
$$

This concludes the proof of the fact that $\|\cdot\|_{L, m, 2}$ are dominated by $\|\cdot\|_{m, 2}$ and $\|\cdot\|_{m, 2}^{\sim}$ norms. 
To show converse domination, we need to prove that

$$
\left\|\nabla^{m} f\right\|_{2} \leq C\left(\|f\|_{2}+\left\|L^{\frac{m}{2}} f\right\|_{2}\right)
$$

with some constant $C \in(0, \infty)$ independent of $f$. The cases $m \leq 3$ was already proven in Theorem 10. We assume by induction that this holds for all $i$ such that $3 \leq i \leq m-$ 1. Applying the case $m=2$ to $\nabla^{m-2} f$, we have $\left\|\nabla^{m} f\right\|_{2} \leq C\left\|L \nabla^{m-2} f\right\|_{2}$. Using the induction assumption, we have

$$
\begin{aligned}
\left\|L \nabla^{m-2} f\right\|_{2} & \leq\left\|\nabla^{m-2} L f\right\|_{2}+\left\|\left[L, \nabla^{m-2}\right] f\right\|_{2} \\
& \leq C\left(\|L f\|_{2}+\left\|L^{\frac{m}{2}} f\right\|_{2}+\left\|\left[L, \nabla^{m-2}\right] f\right\|_{2}\right)
\end{aligned}
$$

For the last term, by Lemma 7, with some constant $\hat{C}$, we have

$$
\left\|\left[L, \nabla^{m-2}\right] f\right\|_{2} \leq \hat{C} \sum_{j=2}^{m}\left\|\nabla^{j} U \nabla^{m-j} f\right\|_{2}
$$

For each $2 \leq j \leq m-1$, using Theorem 12, for any $\delta>0$ with some constant $C_{\delta} \in(0, \infty)$, we have

$$
\left.\left\|\nabla^{j} U \nabla^{m-j} f\right\|_{2}^{2} \leq \delta\left\|\nabla^{m} f\right\|_{2}^{2}+C_{\delta}\|f\|_{2}^{2}\right)
$$

Combining everything together, we arrive at

$$
\left\|\nabla^{m} f\right\|_{2} \leq C \delta\left\|\nabla^{m} f\right\|_{2}^{2}+D(\delta)\left(\|f\|_{2}^{2}+\left\|L^{\frac{m}{2}} f\right\|_{2}^{2}\right)
$$

Choosing $\delta=\frac{1}{2 C}$, we arrive at the desired result.

Next we study the case $p>2$. Before that, let us note that the seminorm $\left\|L^{\frac{j}{2}} f\right\|_{p}$ has similar properties as $\left\|\nabla^{j} f\right\|_{p}$. First of all for $p=2$ using spectral theory one can show that, for the non negative selfadjoint operator $L$, any $\delta \in(0, \infty)$ with some constant $C_{\delta} \in(0, \infty)$, one has

$$
\left\|L^{\frac{k}{2}} f\right\|_{2} \leq \delta\left\|L^{\frac{m}{2}} f\right\|_{2}+C_{\delta}\|f\|_{2}
$$

Since in our context $-L$ is a Markov generator, for any $p \in[1, \infty)$ and any $\lambda \in(0, \infty)$, we have the following property of resolvent

$$
\left\|(\lambda+L)^{k} f\right\|_{p} \leq\|f\|_{p}
$$

and hence

$$
\left\|(\lambda+L)^{-k} f\right\|_{p} \leq\|f\|_{p}
$$

Theorem 14 Suppose condition $\left(\boldsymbol{A}_{m}\right)$ holds. Then for any $p \in[2, \infty)$,

$$
\left\|\nabla^{m} f\right\|_{p} \leq C\left(\|f\|_{p}+\left\|L^{\frac{m}{2}} f\right\|_{p}\right)
$$

Proof We prove this by induction. For $m=2$,

$$
\left\|\nabla^{2} f\right\|_{p} \leq C\left(\|f\|_{p}+\|L f\|_{p}\right)
$$

and for $m=1$

$$
\|\nabla f\|_{p} \leq C\left(\|f\|_{p}+\left\|L^{\frac{1}{2}} f\right\|_{p}\right)
$$

we refer to Theorem 2.1 in [34], (see also nice review in [13] concerning O-U semigroups and references therein). Suppose

$$
\left\|\nabla^{m} f\right\|_{p} \leq C\left(\|f\|_{p}+\left\|L^{\frac{m}{2}} f\right\|_{p}\right)
$$


then by inductive assumption, Minkowski inequality and Theorem 3, we get

$$
\begin{aligned}
\left\|\nabla^{m+2} f\right\|_{p} & \leq C\left(\left\|L\left(\nabla^{m} f\right)\right\|_{p}+\left\|\nabla^{m} f\right\|_{p}\right) \\
& \leq C_{1}\left(\left\|\left[L, \nabla^{m}\right] f\right\|_{p}+\left\|\nabla^{m}(L f)\right\|_{p}+\|f\|_{p}+\left\|L^{\frac{m}{2}} f\right\|_{p}\right) \\
& \leq C_{2}\left(\left\|\left[L, \nabla^{m}\right] f\right\|_{p}+\left\|L^{\frac{m+2}{2}} f\right\|_{p}+\|L f\|_{p}+\|f\|_{p}+\left\|L^{\frac{m}{2}} f\right\|_{p}\right)
\end{aligned}
$$

with some constants $C_{1}, C_{2} \in(0, \infty)$ independent of $f$. In order to deal with the first term, we use the commutator Lemma 7 and the revised Adam's inequality of Theorem 12, to get for any $\epsilon>0$,

$$
\begin{aligned}
\left\|\left[L, \nabla^{m}\right] f\right\|_{p} & \leq C \sum_{i=1}^{m+1}\left\|\nabla^{i+1} U \nabla^{m-i+1} f\right\|_{p} \\
& \leq C \sum_{i=1}^{m+1}\left(\epsilon\left\|\nabla^{m+2} f\right\|_{p}+K(\epsilon)\left\|\nabla^{m-i+1} f\right\|_{p}\right) \\
& \leq C\left(\epsilon\left\|\nabla^{m+2} f\right\|_{p}+\|f\|_{p}\right)
\end{aligned}
$$

Taking $\epsilon<\frac{1}{2 C}$, combining the last estimates, we obtain

$$
\left\|\nabla^{m+2} f\right\|_{p} \leq C\left(\|f\|_{p}+\left\|L^{\frac{m+2}{2}} f\right\|_{p}\right)
$$

Theorem 15 Suppose condition $\left(\boldsymbol{A}_{m}\right)$ holds. Then for any $p \in[2, \infty)$,

$$
\left\|L^{\frac{m}{2}} f\right\|_{p} \leq C\left(\|f\|_{p}+\left\|\nabla^{m} f\right\|_{p}\right)
$$

Proof First of all we note that

$$
\begin{aligned}
\|L f\|_{p} & \leq \sum_{j}\left\|\nabla_{j}^{2} f\right\|_{p}+\|(\nabla U) \cdot \nabla f\|_{p} \\
& \leq \sum_{i, j}\left\|\nabla_{i} \nabla_{j} f\right\|_{p}+\sum_{j}\left\|\left(\nabla_{j} U\right) \nabla_{j} f\right\|_{p}
\end{aligned}
$$

thus

$$
\|L f\|_{p} \leq C_{1, p}\|f\|_{2, p}
$$

Next assume that for any $1 \leq m \leq k$ we have the following bound

$$
\left\|L^{m} f\right\|_{p} \leq C_{m, p}\|f\|_{2 m, p}
$$

Then, using the inductive assumption definition of $L$ and the norms $\|\cdot\|_{m, p}$, we have

$$
\begin{aligned}
\left\|L^{m+1} f\right\|_{p} & =\left\|L^{m}(-\Delta+\nabla U \cdot \nabla) f\right\|_{p} \\
& \leq C_{m, p}\left(\|(\Delta) f\|_{2 m, p}+\|(\nabla U \cdot \nabla) f\|_{2 m, p}\right) \\
& \leq C_{m, p}\|f\|_{2(m+1), p}+C_{m, p}\|(\nabla U \cdot \nabla) f\|_{2 m, p}
\end{aligned}
$$

Combining all that we with some constant $C_{m+1, p} \in(0, \infty)$, we have

$$
\left\|L^{m+1} f\right\|_{p} \leq C_{m+1, p}\|f\|_{2(m+1), p}
$$


and hence by principle of mathematical induction for all $m \in \mathbb{N}$.

\section{Higher Order Decay to Equilibrium}

In this section we discuss the decay to equilibrium for higher order gradients. First we show under general assumptions utilised in the previous section we have the exponential decay to equilibrium of higher order gradients.

For a semigroup $P_{s} \equiv e^{s L}$ generated by $L=\Delta-\nabla U \cdot \nabla$, we set $f_{t} \equiv P_{t} f$

Suppose Poincaré inequality holds

$$
m_{0} \mu(f-\mu f)^{2} \leq \mu|\nabla f|^{2}
$$

Then for $n \geq 0$, we have

$$
\frac{d}{d t} \mu\left(L^{n} f_{t}\right)^{2}=-2 \mu\left|\nabla L^{n} f_{t}\right|^{2} \leq-2 m_{0} \mu\left(L^{n} f_{t}-\mu L^{n} f_{t}\right)^{2}
$$

and so

$$
\mu\left(L^{n} f_{t}-\mu L^{n} f_{t}\right)^{2} \leq e^{-2 m_{0} t} \mu\left(L^{n} f-\mu L^{n} f_{t}\right)^{2} .
$$

Then under the conditions of Theorem 11 , there exists a constant $C \in(0, \infty)$ such that for $k \in \mathbb{N}$

$$
\mu\left(\nabla^{k} f_{t}\right)^{2} \leq C\left\|f_{t}-\mu f\right\|_{L, \frac{k}{2}, 2}^{2} \leq e^{-2 m_{0} t} C\left\|f_{t}-\mu f\right\|_{L, \frac{k}{2}, 2}^{2}
$$

and hence we conclude with the following result.

Theorem 16 Suppose $U$ is locally bounded, $|\nabla U(x)| \rightarrow|x| \rightarrow \infty \infty$ and it satisfies assumption $\left(\boldsymbol{A}_{k}\right)$.

Suppose there exists $m_{0} \in(0, \infty)$ such that

$$
m_{0} \mu(f-\mu f)^{2} \leq \mu\left(|\nabla f|^{2}\right)
$$

Then there exists a constant $C^{\prime} \in(0, \infty)$ such that for any $t \in(0, \infty)$, we have

$$
\mu\left(\nabla^{k} f_{t}\right)^{2} \leq C^{\prime} e^{-2 m_{0} t}\left(\mu\left(\nabla^{k} f\right)^{2}+\mu(f-\mu f)^{2}\right)
$$

Since under our conditions there exists a constant $c_{k, 2} \in(0, \infty)$

$$
\mu\left(f-M_{k, 2} f\right)^{2} \leq c_{k, 2} \mu\left(\left|\nabla^{k} f\right|^{2}\right)
$$

so there exists $C^{\prime \prime} \in(0, \infty)$ such that

$$
\mu\left(\nabla^{k}\left(f-M_{k, 2}\right)_{t}\right)^{2} \leq C^{\prime \prime} e^{-2 m_{0} t} \mu\left(\nabla^{k} f\right)^{2}
$$

o

Motivated by the O-U case where it is possible to obtain explicit control for multiparticle case, in the rest of this section we discuss some heuristic arguments which suggest that in a class of models a stronger decay can be expected. First of all we have the following relation.

$$
\begin{array}{r}
\partial_{s} P_{t-s}\left|\nabla^{k} f_{s}\right|^{2}=P_{t-s}\left(-L\left|\nabla^{k} f_{s}\right|^{2}+2 \nabla^{k} f_{s} \cdot \nabla^{k} L f_{s}\right) \\
=P_{t-s}\left(\left(-L\left|\nabla^{k} f_{s}\right|^{2}+2 \nabla^{k} f_{s} \cdot L \nabla^{k} f_{s}\right)+2 \nabla^{k} f_{s} \cdot\left[\nabla^{k}, L\right] f_{s}\right)
\end{array}
$$


Now recall that, since $L$ is a Markov generator, we have

$$
-L\left|\nabla^{k} f_{s}\right|^{2}+2 \nabla^{k} f_{s} \cdot L \nabla^{k} f_{s}=-2\left|\nabla^{k+1} f_{s}\right|^{2}
$$

Next as a corollary of Lemma 7, we get

$$
\left[\nabla_{j_{1}, \ldots, j_{k}}^{k}, \nabla_{l} U\right]=\sum_{r=1}^{k} \sum_{\mathbf{j}_{r}, \mathbf{i}_{k-r}}\left(\nabla_{\mathbf{j}_{r}}^{r} \nabla_{l} U\right) \nabla_{\mathbf{i}_{k-r}}^{k-r}
$$

with corresponding partitions $\mathbf{j}_{r} \equiv\left\{j_{n_{1}}, . ., j_{n_{r}}\right\}$ and $\mathbf{i}_{k-r}\left\{j_{n_{r+1}}, . ., j_{n_{k}}\right\}$ of $j_{1}, . ., j_{k}$, of cardinality $r$ and $k-r$, respectively, and hence

$$
\left[\nabla_{j_{1}, \ldots, j_{k}}^{k}, L\right]=-\sum_{r=1}^{k} \sum_{\mathbf{j}_{r}, \mathbf{i}_{k-r}}\left(\nabla_{\mathbf{j}_{r}}^{r} \nabla_{l} U\right) \nabla_{\mathbf{i}_{k-r}}^{k-r} \nabla_{l}
$$

From this we obtain

$$
\begin{array}{r}
\partial_{s} P_{t-s}\left|\nabla^{k} f_{s}\right|^{2}=P_{t-s}\left(-2\left|\nabla^{k+1} f_{s}\right|^{2}-2 k \nabla_{j} \nabla^{k-1} f_{s} \cdot\left(\nabla_{j} \nabla_{i} U\right) \nabla_{i} \nabla^{k-1} f_{s}\right. \\
\left.-2 \nabla^{k} f_{s} \cdot \sum_{r=2}^{k} \sum_{\mathbf{j}_{r}, \mathbf{i}_{k-r}}\left(\nabla_{\mathbf{j}_{r}}^{r} \nabla_{l} U\right) \nabla_{\mathbf{i}_{k-r}}^{k-r} \nabla_{l} f_{s}\right) .
\end{array}
$$

Taking expectations and applying quadratic inequalities, after some rearrangement we get

$$
\begin{aligned}
& \partial_{s} \mu\left|\nabla^{k} f_{s}\right|^{2}+2 k \mu\left(\nabla_{j} \nabla^{k-1} f_{s} \cdot\left(\nabla_{j} \nabla_{i} U\right) \nabla_{i} \nabla^{k-1} f_{s}\right)-2 k \varepsilon \mu\left|\nabla^{k} f_{s}\right|^{2} \\
& \leq \frac{1}{2 k \varepsilon} \sum_{r=2}^{k} \sum_{\mathbf{j}_{r}, \mathbf{i}_{k-r}} \mu\left(\left|\nabla_{\mathbf{i}_{k-r}}^{k-r} \nabla_{l} f_{s}\right|^{2}\left|\nabla_{\mathbf{j}_{r}}^{r} \nabla_{l} U\right|^{2}\right) .
\end{aligned}
$$

Hence we see that first necessary condition for a faster decay is the following: There exists $m \in(0, \infty)$ such that

$$
m \mu\left|\nabla^{k} f\right|^{2} \leq \mu\left(\nabla_{j} \nabla^{k-1} f_{s} \cdot\left(\nabla_{j} \nabla_{i} U\right) \nabla_{i} \nabla^{k-1} f_{s}\right) .
$$

Naturally one can satisfy this condition for strictly convex $U$. To complete the estimates on the decay to equilibrium one needs to estimate the right hand side of Eq. 80 which could be achieved with the techniques developed by us in the earlier sections possibly with additional restriction on $U$. This will be studied in more detail elsewhere.

Data Statement : The research does not include any data.

Open Access This article is licensed under a Creative Commons Attribution 4.0 International License, which permits use, sharing, adaptation, distribution and reproduction in any medium or format, as long as you give appropriate credit to the original author(s) and the source, provide a link to the Creative Commons licence, and indicate if changes were made. The images or other third party material in this article are included in the article's Creative Commons licence, unless indicated otherwise in a credit line to the material. If material is not included in the article's Creative Commons licence and your intended use is not permitted by statutory regulation or exceeds the permitted use, you will need to obtain permission directly from the copyright holder. To view a copy of this licence, visit http://creativecommons.org/licenses/by/4.0/.

\section{References}

1. Adams, R.A.: General logarithmic Sobolev inequalities and Orlicz embeddings. J. Func. Anal. 34, 292303 (1979). https://doi.org/10.1016/0022-1236(79)90036-3 
2. Adams, R.A.: Sobolev spaces. Academic Press, Cambridge (1975)

3. Aida, S.: An estimate of the gap of spectrum of Schrödinger operators which generate hyperbounded semigroups. J. Funct. Anal. 185, 474-526 (2001)

4. Ané, C., Blachère, S., Chafai, D., Fougères, P., Gentil, I., Malrieu, F., Roberto, C., Scheffer, G.: Sur les inégalités de Sobolev logarithmiques, Panor Synthèses, vol. 10. S.M.F., Paris (2000)

5. Auscher, P., Tchamitchian, P.: Square root problem for divergence operators and related topics Astérisque, tome 249. http://www.numdam.org/item?id=AST_1998_249_R1_0 (1998)

6. Bakry, D., Gentil, I., Ledoux, M.: Analysis and Geometry of Markov DiffusionOperators, Grundlehren der mathematischenWissenschaften, vol. 348, p. 552. Springer, Berlin (2014)

7. Bauerschmidt, R., Bodineau, T.: Log-Sobolev inequality for the continuum sine-Gordon model. https:// arxiv.org/pdf/1907.12308.pdf (2020)

8. Barthe, F., Roberto, C.: Modified logarithmic Sobolev inequalities on R. Potential Anal. 29, 167 (2008). https://doi.org/10.1007/s11118-008-9093-5

9. Barthe, F., Cattiaux, P., Roberto, C.: Interpolated inequalities between exponential and Gaussian, Orlicz hypercontractivity and application to isoperimetry. Rev. Mat. Iberoamericana 22, 993-1067 (2006)

10. Bobkov, S.G., Götze, F.: Exponential integrability and transportation cost related to logarithmic Sobolev inequalities. J. Funct. Analysis. 1, 1-28 (1999)

11. Bobkov, S.G., Zegarlinski, B.: Entropy bounds and isoperimetry. Mem. Amer. Math. Soc. 176(829), $\mathrm{x}+69(2005)$

12. Bobkov, S.G., Zegarlinski, B.: Distributions with slow tails and ergodicity of Markov semigroups in infinite dimensions. In: Laptev, A. (ed.) Around the Research of Vladimir Maz'ya I. International Mathematical Series, vol. 11 (2010). https://doi.org/10.1007/978-1-4419-1341-8_2

13. Bogachev, V.I.: Ornstein-Uhlenbeck operators and semigroups. Russ. Math. Surv. 73, 191 (2018)

14. Bolley, F.: I. Gentil, Phi-entropy inequalities for diffusion semigroups. J. Math. Pures Appl. 93, 449-473 (2010)

15. Cipriani, F.abio.: Sobolev-Orlicz imbeddings, weak compactness, and spectrum. J. Func. Analysis 177, 89106 (2000). https://doi.org/10.1006/jfan.2000.3633

16. Davies, E.B., Gross, L., Simon, B.: Hypercontractivity: A bibliographic review. In: Albeverio, S. (ed.) Proceedings Hoegh-Krohn Memorial Conference (1992)

17. Davies, E.B., Simon, B.: J. Func. Analysis 59. 335-395 Ultracontractivity and the heat kernel for Schrödinger operators and Dirichlet Laplacians. https://doi.org/10.1016/0022-1236(84)90076-4 (1984)

18. Deuschel, J.D., Stroock, D.W.: Hypercontractivity spectral gap of symmetric diffusions with applications to the stochastic ising model. J. Funct Analysis 92, 48 (1990)

19. Chafaï, D.: Entropies, convexity, and functional inequalities on Phi-entropies and Phi-Sobolev inequalities. J. Math. Kyoto Univ. 44(2), 325-363 (2004). https://doi.org/10.1215/kjm/1250283556, arXiv:math/0211103v2

20. Drábek, P., Kufner, A., Nicolosi, F.: Quasilinear elliptic equations with degenerations and singularities, vol. 5. Walter de Gruyter, Berlin (2011)

21. Wang, F.-Y.: Orlicz-Poincaré inequalities. Proc. Edinburgh Math. Soc. 51(02). https://doi.org/10.1017/ S0013091506000526 (2006)

22. Guionnet, A., Zegarlinski, B.: Lectures on logarithmic Sobolev inequalities. In: Séminaire de Probabilités, XXXVI, volume 1801 of Lecture Notes in Math., pp. 1-134. http://www.numdam.org/article/ SPS_2002__36__1_0.pdf

23. Hebisch, W., Zegarlinski, B.: Coercive inequalities on metric measure spaces. J. Func. Analysis 258, 814-851 (2010). https://doi.org/10.1016/j.jfa.2009.05.016

24. Holley, R., Stroock, D.: Logarithmic Sobolev inequalities and stochastic Ising models. J. Statist. Phys. 46(5-6), 1159-1194 (1987)

25. Inglis, J., Neklyudov, M., Zegarlinski, B.: Ergodicity for infinite particle systems with locally conserved quantities. Infin. Dimens. Anal. Quantum Probab. Relat. Top. 15(1), 1250005 (2012). 28 pp., ISSN:02190257, https://doi.org/10.1142/S0219025712500051

26. Kontis, V., Ottobre, M., Zegarlinski, B.: Long-andshort-time behaviour of hypocoercive- type operators in infinite dimensions: An analytic approach. Infin. Dimens. Anal. Quantum. Probab. Relat. Top. https://doi.org/10.1142/S0219025717500151, arXiv:1306.6453 (2017)

27. Kontis, V., Ottobre, M., Zegarlinski, B.: Markov semigroups with hypocoercive-type generator in Infinite Dimensions I : Ergodicity and Smoothing. J. Funct. Anal. 270, 3173-3223 (2016). https://doi.org/10. 1016/j.jfa.2016.02.005, arXiv:1306.6452

28. Krasnosel'skii, M.A.: Ya. B. Rutickii - Convex Functions and Orlicz Spaces. P Noordhoff Groningen (1961)

29. Martinelli, F.: On the two-dimensional dynamical Ising model in the phase coexistence region. J. Stat. Phys. 76, 1179-1246 (1994) 
30. Masmoudi, N., Sani, F.: Higher order Adams' inequality with the exact growth condition. Commun. Contemp. Math. Vol. 20, No. 6, 1750072 (2018). https://doi.org/10.1142/S0219199717500729

31. Rao, M.M., Ren, Z.D.: Theory of Orlicz Spaces Pure and Applied Mathematics, vol. 146. Marcel Dekker, New York (1991)

32. Roberto, C., Zegarlinski, B.: Orlicz-Sobolev inequalities for sub-Gaussian measures and ergodicity of Markov semi-groups. J. Func Anal. 243, 28-66 (2007)

33. Rosen, J.: Sobolev inequalities for weight spaces and supercontractivity. Trans. Am. Math. Soc. 222, 367-376 (1976). American Mathematical Society. http://www.jstor.org/stable/1997677

34. Li, X.iang.D.ong.: Riesz transforms for symmetric diffusion operators on complete Riemannian manifolds. Rev. Mat. Iberoamericana 22(2), 591-648 (2006)

35. Wang, F.-Y.: Functional inequalities for empty essential spectrum. J. Funct. Anal. 170, 219245 (2000)

36. Won, Y.ong.S.ul.: Analysis of linear and nonlinear dissipative systems. PhD Thesis Imperial College London (2018)

37. Zegarlinski, B.: Entropy bounds for Gibbs measures with non-Gaussian tails. J. Func. Analysis 187, 368-395 (2001). https://doi.org/10.1006/jfan.2001.3808

38. Ziemer, W.P.: Weakly differentiable functions :Sobolev spaces and functions of bounded variation, vol. 120. Springer Science \& Business Media, Berlin (2012)

Publisher's Note Springer Nature remains neutral with regard to jurisdictional claims in published maps and institutional affiliations.

\section{Affiliations}

\section{Yifu Wang $^{1}$ (D) . Boguslaw Zegarlinski ${ }^{1}$}

Yifu Wang

yifu.wang17@imperial.ac.uk

1 Department of Mathematics, Imperial College London, 180 Queen's Gate, London, SW7 2AZ, UK 